\title{
A systematic investigation of cycle number, temperature and electric field strength effects
}

\section{on Si anode}

\author{
Qihong Fanga, Qiong Wanga, Jia Lia ${ }^{a^{*}}$, Eenze Chen ${ }^{\mathrm{a}}$, Bin Liu ${ }^{\mathbf{k}}$, Pihua Wenc \\ a State Key Laboratory of Advanced Design and Manufacturing for Vehicle Body, Hunan University, Changsha,
} 410082, PR China

${ }^{b}$ State Key Laboratory for Powder Metallurgy, Central South University, Changsha, 410083, P. R. China

c School of Engineering and Material Sciences, Queen Mary, University of London, London E1 4NS, UK

* Corresponding author. E-mail address: lijia123@hnu.edu.cn (J. Li); binliu@csu.edu.cn (B. Liu).

\begin{abstract}
Cycling number, crystal orientation, temperature and electric field strength play key roles in affecting the capacity and cycling ability of Li-ion battery. However, the detailed dynamic and continuous process of capacity decline mechanism from above factors need to be further understood at nanoscale. Herein, we report deformation behavior and microstructure evolution of Si anode under electric field using molecular dynamics simulations. The results show larger cycling number and electric field strength cause larger volume expansion of Si electrode, leading to the capacity loss and the reducing cycling ability as well as the decreasing structural stability. The phase transformation from diamond cubic structure to body-centred tetragonal structure and the amorphous formation depend on lithiated and delithiated depths. The crystal orientation [100] Si anode produces the volume expansion owing to a large number of nanoscale void. The various temperatures strongly affect the number and radius of nanovoid. The associated transition in the nanovoid nucleation due to the involuntary diffusion process is driven by larger cycling number or electric field strength, resulting in the irreversible capacity loss of Li-ion battery. An established analytical model suggests that diffusion-induced stress not only relies on the position of $\mathrm{Si}$ anode but depends upon the cycling time.
\end{abstract}

Keywords: Silicon electrode; Cycling number; Temperature; Electric field strength; Molecular dynamics; 


\section{Graphical abstract}

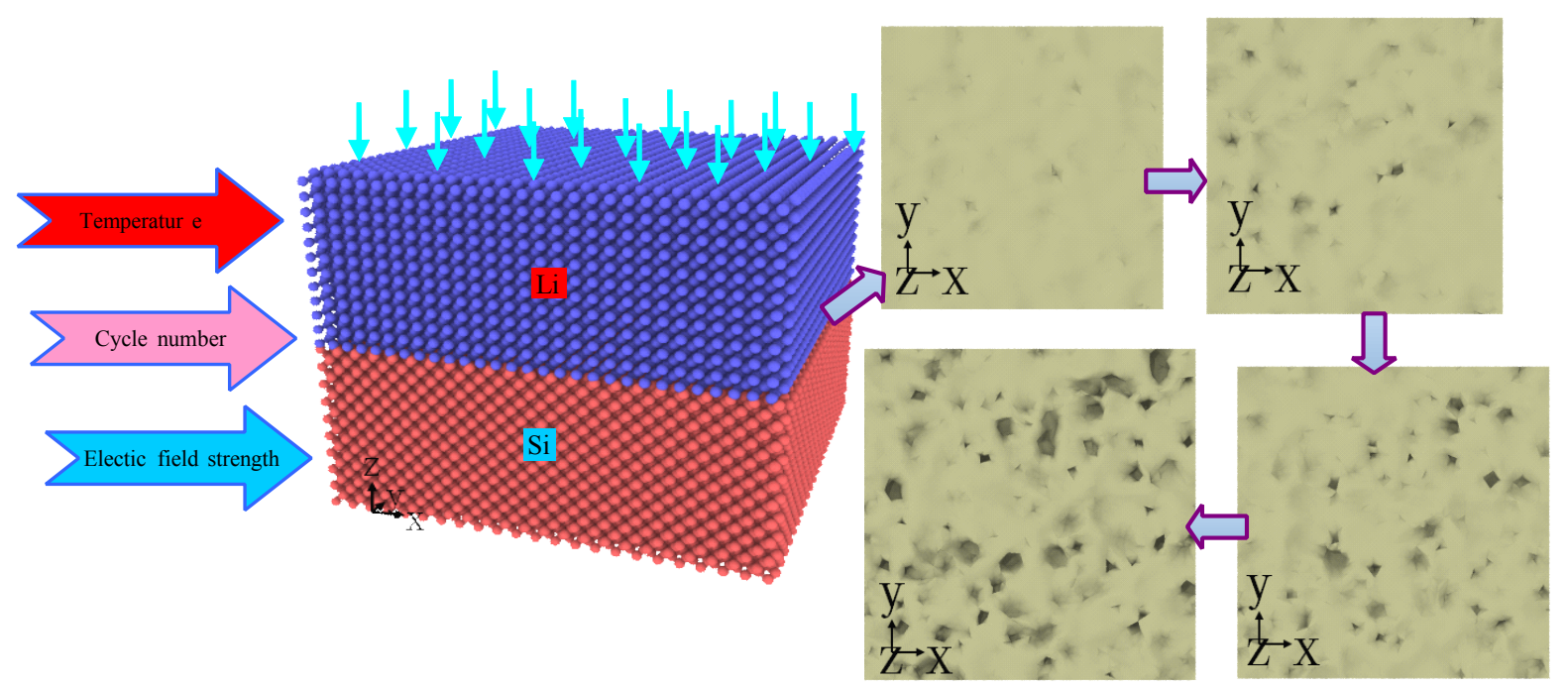

\section{Introduction}

Li-ion batteries have attracted extensive research interest in recent years due to reports of high energy density and excellent operating voltage, which have gotten broad applications in mobile electronic devices and electric vehicles [1-3]. In most cases, Li-ion batteries have superior rate capability but at the expense of capacity and cyclic performance $[4,5]$, which considerably limits the life and practical utility of Li-ion battery. The failure mechanism of Li-ion battery depends on the dominant aging modes [6-10], such as extremely high gradient stress [6,7], formation and growth of solid-electrolyte interphase (SEI) [8, 9], and cracking [10]. More intriguingly, the electrochemical and mechanical properties of Li-ion battery would be influenced when the amorphous Si electrodes undergo the large plastic deformation [11-13]. Therefore, it is strongly suggested that further study on the failure mechanisms during cycling are of great necessity and finally achieve the purpose of prolonging the Li-ion battery life by reducing and controlling the its plastic behavior.

Some experiments $[14,15]$ show that the loss of structural integrity to reduce the electrical conductance results in the fading of the capacity of Li-ion battery during cycling. Some theoretical modelings show the elastic-plasticity behavior of $\mathrm{Li}_{x} \mathrm{Si}$ alloys described by the continuum mechanics theories, and reveal the stress-gradient effects during the Li-ion diffusion process $[16,17]$. Diffusion-induced stress computed by the analytical expression is investigated for nanowire [18], cylindrical modeling [19], plate modeling [20], and spherical modeling [21, 22]. The deformation behavior of amorphous LixSi alloys have been well explained 
by some atomic simulations, such as density functional theory (DFT) calculations and molecular dynamics (MD) simulations [23-25]. Using the reactive-force-field (ReaxFF) potential, Ostadhossein et al. [24] studied the lithiation process of the crystal Si nanowires. They found that Li insertion into interlayer spacing between two adjacent (111) planes results in the amorphization, which is in good agreement with experimental observations. By the modified embedded atomic method (MEAM), Cui et al. [25] predicted the mechanical properties of crystal and amorphous $\mathrm{Li}_{x} \mathrm{Si}$ alloys, such as Young's modulus, yield strength and Poisson's ratio. However, all the studies are concentrated on the plastic deformation mechanisms of Si electrode, no systematic research has been dedicated to the effects of crystal orientation, cycling number, temperature and electric field strength on the nanoscale deformation behavior in the Si anode during cycling.

The manuscript is organized as follows: Section 2 contains the details of MD simulations of lithiation and delithiation. Section 3 discusses the MD results, which are compared with previous experimental observations and atomic simulation results. The effects of crystal orientation, cycling number, temperature, and electric field strength on deformation behavior and structural stability of Si anode are investigated with the aid of MD simulation. In addition, the analytical model on the diffusion-induced stress is established. Our conclusions are shown in Section 4. Our work would be able to provide a plastic deformation and structural stability during the charge-and-discharge cycle. Although the Li-ion battery failure does not originate from a single reason, the current studies help us further understand the nanoscale failure mechanism of Si anode and improve the Li-ion battery life.

\section{Simulation Details}

Using large-scale atomic/molecular massively parallel simulator (LAMMPS) [26], the $\mathrm{Si}$ anode and Li electrode under electric field are performed by MD simulations, which are used to study solute diffusion driven by the electric field $[27,28]$, as shown in Fig. 1a. The MD model is build by the single-crystal Li electrode and single-crystal Si anode. The every Li-ion has a unit positive charge [28], and the every Si atom maintains neutral. The dimension of Si electrode consisting of $43,560 \mathrm{Si}$ atoms is $12.2 \times 12.2 \times 5 \mathrm{~nm}^{3}$, and that of Li electrode consisting of $42,875 \mathrm{Li}$-ions is $12.2 \times 12.2 \times 5 \mathrm{~nm}^{3}$. The red and blue spherical particles represent Li-ions and $\mathrm{Si}$ atoms, respectively. The crystal orientations of the $\mathrm{Li}$ and $\mathrm{Si}$ electrodes are [100] along $x$ direction, [010] along $y$ direction, and [001] along $\mathrm{z}$ direction. The lithiation is performed under a constant electric-field strength, while the delithiation is performed under a same constant electric-field strength with opposite direction. According to the work [29], the electric current density $I$ and electric field strength $E$ can be expressed by $E=\| / \sigma$, where $\sigma$ is the electrical conductivity. During the charging and discharging of Li-ion 
battery, the electric current density is $=0.5 \mathrm{~mA} / \mathrm{cm}^{2}$ [30]. The electrical conductivity of $\mathrm{Si}$ is $\sigma=4 \times 10^{-5} \mathrm{~S} / \mathrm{cm}$ [31]. Hence, the electric field can be calculated $E=1.25 \mathrm{~V} / \mathrm{cm}$. In order to improve the calculation efficiency combined with the reasonable computation time, a higher electric field $E=1 \mathrm{~V} / \AA$ is used to achieve the charging and discharging process in Li-ion battery. Periodic boundary conditions are imposed in both the $x$ and $y$ directions, and the free surface is used to the $z$ direction [28]. The MEAM potential is used to describe the atomic interactions $[25,28]$, which can be capable of accurately predicting the mechanical properties of Li-ion battery over nanometer scales $[7,25,28]$. In addition, the electrostatic interactions between the intercalated Li-ions account for the Coulomb interactions for the MD simulation system.

According to the Maxwell-Boltzmann distribution, the velocities of all Li-ions and Si atoms are randomly given. The temperature is maintained at target temperatures, such as 100, 200, 300, 500 and $800 \mathrm{~K}$, via Nose-Hoover thermostat [28]. In the all simulation cases, the time step is set to 1 fs, and the isobaric-isothermal canonical ensemble (NPT) dynamics is performed. During the first charging-and-discharging cycle, the simulations are divided into three stages as followed: firstly, at the relaxation stage, the system is kept at target temperature for 100 ps to achieve an equilibrium state; secondly, at lithiation stage Li-ion begins to insert into Si electrode under the given electric field along the negative z-axis direction until initial Li-ions reach the end of Si electrode. To conduct the every step of lithiation process, the electric field is applied in every $10 \mathrm{fs}$, and then the system is relaxed to an equilibrium state for 100 ps; finally, at delithiation stage Li-ion begins to extract into Si electrode under the given electric field along the z-axis inverse direction until all Li-ions leave the upper surface of Si electrode. To conduct the every step of the delithiation process, the electric field with inverse direction is applied in every $10 \mathrm{fs}$, and then the system is relaxed to an equilibrium state for 100 ps. For next charging-and-discharging cycle, a similar way related to the electric field acts on the electrodes. Although the massive MD calculations successfully offer important insights into the relationship between microstructure and properties based on the high-performance computing, notwithstanding the fact that the time scale of MD simulation on large-scale systems is typically less than 1000 ps. Hence, the current simulated cycling of Li-ion battery at a very short time period is mainly focused on the microstructure-dominated electrochemical performance, to reveal the deformation and failure mechanism during cycling. Moreover, this study could gain further understanding of real cycling behavior to guide the design and optimalize the structure in Li-ion battery.

In this simulation, the open visualization tool (OVITO) software is used to visualize the LAMMPS output data for the charging-and-discharging process [32]. The evolution and distribution of the possible phases 
during cycling are discussed in terms of coordination number $(\mathrm{CN})$ and radial distribution function (RDF).

\section{Results and discussion}

\subsection{Lithiation and delithiation phenomenon}

The Li-ion diffusion processes in the $\mathrm{Si}$ anode have been carefully studied by experiments, theoretical models and simulations [16-22, 33-38]. However, most of these experiential, theoretical and simulated results are focused on the structure and mechanical properties of the final status of $\mathrm{Li}_{x} \mathrm{Si}$ alloys [36-38], which could not model diffusion-induced strain upon electrochemical lithiation and delithiation. On the other hand, the detailed atomistic mechanism of surface morphology and phase transformation as well as structural stability in Si electrode during cycling are not fully reported. To understand the experimental observation of one-dimensional fast diffusion of Li-ion under electric field, it is necessary to investigate the nanoscale plastic deformation. In the present study, the deformation behavior of Li and Si electrodes in first cycling is studied via MD simulations. However, the current MD simulations cannot capture SEI layer formation and growth, which play a critical role in the electrochemical performance of Si anode, owing to the SEI layer composed of inorganic and organic electrolyte decomposition products $[39,40]$. Hence, the formation and growth of SEI layer is not discussed during charging and discharging.

With increasing the lithiatied depth, Fig. 2a-d shows the evolution of structural characterization in Li electrode and Si electrode. The lithiatied depth is defined as the distance between $\mathrm{Si}$ atom at the Si-electrode top and Li-ion at the Li-electrode bottom (see, Fig. 2a). To explain the structural evolution of Si anode during lithiation, we use CN method with OVITO software to identify crystal structure of Si-Si under different lithiated depths [41-43], as shown in Fig. 2e-h. In the initial lithiated stage, Si anode has a relatively complete crystal structure (see, Fig. 2e). In addition, the increasing lithiated depths cause a gradual transition from crystal to amorphous Si identified in Fig. 2e-h. Fig. 2i-l presents the corresponding evolution of surface morphology during lithiation process. It can be found in Fig. $3 \mathrm{~b}$ that the radius and number of nanovoid nucleated from the free surface of Si anode continuously increase with the increasing of lithiated depth. In particular, the large tensile stress built up in Si anode leads to the cracking of lithiated Si layer [44], and then the growth of fresh lithiated Si layer on the crack faces causes further loss of capacity [45]. It is clearly observed that the lithiated depth bending at surface exceeds $3.75 \mathrm{~nm}$. The significant change of the surface inevitably produces the greater stress zone in Si anode, reducing the Li-ion battery performance [46].

The RDF obtained from a diffraction measurement contains the important one-dimensional information, and it provides insight into the structure of lithiated $\mathrm{Si}$ anode. For the perfect single crystal diamond cubic 
structure $\mathrm{Si}$, the three peaks in RDF are $2.35 \AA, 3.85 \AA$ and $4.65 \AA$, respectively, corresponding to the distance $\mathrm{Si}-\mathrm{Si}$ bonds [47]. In Fig. 3a, the similar peaks occur in lithiated Si anode with increasing of lithiated depth. Especially, the first peak in the RDF curve is $2.42 \AA$ for the lithiated depths of $2.5 \mathrm{~nm}$, meaning that the phase transformation occurs from the diamond cubic structure $(\alpha-\mathrm{Si}, \mathrm{Si}-\mathrm{I})$ to the body-centred tetragonal structure ( $\beta$-Si, Si-II) [41-43, 48]. As shown in Fig. 3, the $\beta$-Si has the four nearest neighbours at distance of $2.42 \AA$ and two ones at larger distance of $2.57 \AA[41-43,48]$. There exists a transition from crystal Si to amorphous Si with the continuously increasing thickness of lithiated Si layer, which agrees well with the experimental results obtained from an in-situ TEM during the early stage of cycling [49] (see Figs. 2e-h and 3a).

As the delithiated depth increases, the evolution of deformation behavior and microstructure in the Li and $\mathrm{Si}$ electrodes is shown in Fig. 4. The delithiatied depth is defined as the distance between the upper surface of Si electrode and the upper surface of Li electrode (see, Fig. 4a). As a result, the Si electrodes remain amorphous with the decrease of lithiated Si layer during delithiation process, revealing the occurrence of severe plastic deformation in $\mathrm{Si}$ anode (see, Fig. 4e-h). In addition, the volume of Si electrode during lithiation process increases as the lithiated depth increases; while, it slightly increases with the increasing of delithiated depthes during the delithiation process (see, Fig. 5a). In other words, the Li diffusion proceeds with increasing of the volume change during the charging-and-discharging process, observed clearly in Fig. 5a.

Fig. $5 \mathrm{~b}$ also shows the first peak of $2.42 \AA$ based on the RDF curve, meaning that the body-centred tetragonal structure Si takes place during the delithiation [47]. Hence, a phase transformation from $\alpha$-Si to $\beta$-Si could occur in the delithiation due to the high compressive stress induced by Li-ion diffusion, which produces the volume expansion and interface mismatch (see, Figs. 4 and 5). As the delithiated depth increases, the first peak value in RDF curve continuously increases (see Fig. 5b). To further characterize the structure after the delithiated process, the surface evolution during the delithiation is shown in Fig. 6. The nanovoid in the lithiated surface gradually grows up (see, Fig. 5c), and leads to the recession of Li-ion battery capacity due to reducing the Li-ion passing through cross-sectional area. The critical stress required for the crack nucleation in amorphous electrode decreases compared with that in the single-crystal electrode as a result of the volume expansion to produce the porous Si anode. This phenomenon agrees well with experimental and simulated results, where the high strain regions generated by high Li-ion concentrations induce high stress regions [18-22]. A comparison of Figs. 2 and 6 shows that the nanovoids appear upon lithiation. On the one hand, the relatively low tensile stress could result in the crack/nanovoid of $\mathrm{Si}$ anode as compared to the compressive stress, due to that Si has a higher compressive fracture strength. On the other hand, the lithiation process 
produces the tensile stress, and the delithiation process produces the compressive stress. After lithiation process, the stability of $\mathrm{Si}$ anode declines, which reduces its fracture strength and increases the nanovoid. Hence, the capability of Si-anode Li-ion battery declines after first cycling.

It is well-known that the materials deform inhomogeneously under external loading due to large amount of redundant strain introduced in local region of materials. The strain in the surface and inner regions of Si anode affects the capacity and current density of Si electrode during every cycling. The high strains at the surface and inner of electrode inhibit Li-ion to move towards Si anode. Fig. 7 shows a series of strain distribution in $\mathrm{Si}$ anode for the different lithiated and delithiated depths. The high strain is concentrated in the lithiated Si layers due to the strong interactions between $\mathrm{Si}$ and $\mathrm{Li}$, as illustrated in Figs. 2, 4 and 7. The obvious boundary is observed in amorphous $\mathrm{Si}$ anode [8,9], which is attributed to the formation of lithiated phase. This phenomenon proves the high strain induced by lithiated phase. During delithiated process, the regions of high strain continue to spread toward the upper surface of Si anode, resulting in the maximum strain region in upper surface of Si anode after first charging-and-discharging cycle.

\subsection{Crystal orientation effect}

The crystal orientation can play an important role in determining the cycling properties of Li-ion battery [50]. In order to investigate the crystal orientation effect on the plastic deformation and structural stability, atomic models for other two crystal orientations of [110] and [111] are investigated. Here, the structure and strain distribution of Si anode after lithiated process are shown in Fig. 8. The regions of higher strains occur in [110] and [111] $\mathrm{Si}$ anodes, as compared with [100] $\mathrm{Si}$ anode. The high strain can cause the phase transformation and amorphization of $\mathrm{Si}$ anode. Hence, with further increasing lithiated process, the critical stress required for crack nucleation can drive the nucleation of nanoscale void to reduce the structure stability and cycling performance.

Comparing the simulation results of different crystal orientations in Fig. 8, it can been found that the [111] Si anode produces more nanoscale voids after lithiated process. It is of interest to note that the RDF curves consisting of two peaks are almost the same in Si anode with different crystal orientations (see Fig. 9); it is therefore expected that the volume of Si electrodes would also exhibit a similar crystal orientation effect on the structure stabilities of Si anode. A comparison of volume and RDF from Fig. 9 shows the strong anisotropy of [110] Si anode. In addition, the volume of [100] Si anode is larger than that of [110] and [111] Si anode owing to a large number of small voids formed at surface (see Fig. 9c).

\subsection{Cycling number effect}


Figs. 10 and 11 show the effect of cycling number on the plastic deformation and strain in $\mathrm{Si}$ anode at room temperature of $300 \mathrm{~K}$. The increase of cycling numbers causes the significant changes in the shape of $\mathrm{Si}$ electrode, as shown in Fig. 10. As the cycling numbers increase, high strain region gradually penetrates into the bottom of Si electrode (see, Fig. 11). The high cycling numbers produce a large nonuniform strain in the Si anode, leading to the serious volume expansion (Fig. 12a). According to Fig. 12b, there is a significant difference in the RDF curve. Hence, the cycling numbers which determine the structure stability in Si electrode are a direct indicator of battery life.

Fig. 12b also shows the RDF obtained from MD simulations at cycling numbers of 2, 3, 4, 5, 6 and 7 times. The cycling diffusion induces the high strain and fatigue damage, causing the inevitably capacity loss in Li-ion battery [51]. The significant change of two peaks in RDF curve takes place with the increase of cycling numbers, indicating the occurrence of complex microstructural evolution. In addition, larger cycling numbers cause the larger peaks. Hence, the disordered structure of Si anode strongly depends on cycling numbers. During the lithiated process, the Si-anode structure gradually becomes more disordered and eventually forms the amorphous structure with serious deviation from its original crystal structure, as shown in Figs. 11 and 12. Moreover, there is obvious difference in the surface morphology under various cycling numbers (see Fig. 13). The initial formated void can be healed $[15,51,52]$, and the new voids nucleate in the healed region during cycling. In Fig. 12b, large cycling numbers (large than 5) increase the radius of void and decreases the number of void to accommodate the high strain region (Fig. 11). The current observations provide an atomic-level understanding of cycling number effect on the evolution of void, which reduces the structure stability of Si electrode.

\subsection{Temperature effect}

In future, Li-ion batteries could be used in high temperature or low temperature in order to meet some special environments, such as outer space and deep sea. Hence, the effect of temperature on the deformation behavior and strain in Si electrode is described in Fig. 14a-d. The coefficients of Li-ion diffusion could be affected by some factors such as the temperature, the stress state and the state of charge [53]. It can also be found that the coefficients of Li-ion diffusion are increased at high tensile stress and high temperature [54, 55] To ensure Li-ion diffusion, the systems of MD simulation can reach an equilibrium state at target temperatures for $100 \mathrm{ps}$ after each electric field step of $10 \mathrm{fs}$. Compared to the microstructure at the temperature of $100 \mathrm{~K}$, $200 \mathrm{~K}, 500 \mathrm{~K}$, and $800 \mathrm{~K}$, there are no obvious differences in plastic deformation of Si electrode when Li-ions are fully embedded in Si anode. In order to elucidate the temperature effect on the plastic deformation during 
lithiation, the strain distribution at various temperatures is plotted in Fig. 14e-h. Here, the relative strain is defined as the relationship between the strain from lithiated state and the strain from the balanced state at the target temperature. In Fig. 14e-h, the regions of high strains appear at the top of Si anode for different temperature ranges; the low strain areas occur at the end of Si anode at low temperature, i.e., $100 \mathrm{~K}$. Hence, the serious nonuniform strain distributions take place at low temperature in Si electrode.

Fig. 15a shows the volume of Si electrode firstly increases and then decreases with the increasing of temperature. Although $\mathrm{Li}$ and $\mathrm{Si}$ atoms can have more chances to leave their origin positions at relative higher temperature, the disordered structure of Si anode inhibits the Li-ion diffusion. To obtain better insights about structural changes at various temperatures, the RDF after lithiated process is plotted in Fig. 15b. The first RDF

peak occurs at distance of $2.53 \AA$, deviated from the expected interatomic distance of $2.35 \AA$, which means that there is an amorphous $\mathrm{Si}$ anode. Almost, all curves coincide with each other, indicating that structural changes are weakly dependent on temperature at large deformation. As the temperature of system increases from $100 \mathrm{~K}$ to $800 \mathrm{~K}$, the peaks in RDF curve are slightly decreased with the increasing temperature, in good agreement with DFT study [56]. Hence, the temperature plays a weak role in deformation of Si electrode after cycling. However, the surface morphology of Si electrodes strongly relies on the temperature after cycling, which can be revealed in Fig. 16. The low temperature of $100 \mathrm{~K}$ and the high temperature of $500 \mathrm{~K}$ produce the large void nucleated from free surface, and the high temperature can reduce the number of void owing to the rapid diffusion (see Fig. 15c). However, intermediate temperature promotes crack nucleation [15, 22, 51], dependent upon the competition between $\mathrm{Li}$-ion diffusion rate and volume expansion rate.

\subsection{Electric field strength effect}

Fig. 17 a shows the snapshots of atomistic structure of Si electrode under the electric field strength of 5 $\mathrm{V} / \AA \AA, 2 \mathrm{~V} / \AA \AA, 1 \mathrm{~V} / \AA \AA$, and $0.5 \mathrm{~V} / \AA$. Deformation behavior and strain distribution of Si electrode show a strong dependence on electric field strength (see Fig. 17 b). Under electric field, the Li-ions fast diffuse along one dimensional column, which agrees with experimental result of quasi-one dimensional diffusion channel in disordered Si electrode $[57,58]$. The diffusion induced by electric field strength dominates the Li-ion motion due to that electric field strength depends upon the extent of the electrochemical charge transfer in unit time $[58,59]$. In addition, the higher electric field strength accelerates the Li-ions diffusion and causes the large strain region, which reduces the structural stability of Si anode.

The RDF distribution and the Si-anode volume under different electric field strengths are shown in Fig. 18. It is interesting to note that the first peak in RDF violently decreases with increasing electric field strength, 
observed in Fig. 18b. Therefore, the structural changes of the lithiated Si electrode are great at different electric field strengths. Under the low electric field strength of $0.5 \mathrm{~V} / \AA$, Li-ions could overcome obstacle from lattice resistance of $\mathrm{Si}$ electrode and continue to move into the interior of Si electrode; the lithiated Si anode has enough time to adjust and adapt this strain, resulting in relatively uniform distribution of Si atom and the significant topography change of Si anode (See, Fig. 17). Moreover, the volume of Si electrode would enlarge with the increasing of the electric field strength, due to a large radius of nanovoids. (See, Figs. 18c and 19). The nanovoid formation in interior of amorphous Si electrode appears at the large plastic deformation after cycling (Fig. 19). As shown in Figs. 18c and 19, Li-ion could pass through the Si electrode with high electric field strength, resulting in accelerating the formation of nanovoids. Hence, the weakly stable Si anode leads to battery fail at high voltage cutoff or current [46]. In other word, the speed of Li-ion movement driven by electric field is much greater than the moving speed of Si atoms driven by diffusion. The high electric field strength increases the diffusion stress and contributes to determining the diffusion process during the charge and discharge process.

The above results of MD simulation mentioned is mainly to solve the voltage effect on the intercalation properties. As well known, the SOC, OCV, and concentration of electrolyte also dominate the cycling of Li-ion battery. However, the MD investigation into these factors on the intercalation properties is still very difficult due to the complex structure and composition of Li-ion battery. Hence, the capacity loss and cycling ability as well as structural stability considering the effects of SOC, OCV, and concentration of electrolyte are left in future.

\section{6 diffusion-induced stress of nanofilm silicon electrode}

The above atomic simulations show that the diffusion interface plays a critical role in the deformation behavior and microstructure evolution of lithiated Si electrode. Therefore, for a deeper understanding of the nanoscale interface effect on diffusion-induced stress in the nanoscale Si electrode, we establish a simply analytical model to reveal the relationship between stress and time in the nanofilm electrode. As shown in Fig. 1b, a flux of Li-ions embed into a nanofilm Si electrode with thickness of $h$ during charging. The infinite $x-y$ plane is used to the upper surface of nanofilm Si electrode, and the z-axis is applied to the embedded direction. According to the solute diffusion theory, the Li ion motions driven by gradient solute is regarded as a diffusion process. Because the process of elastic deformation is much faster than atomic diffusion, the mechanical equilibrium during lithiation and delithiation is considered as a static equilibrium model [19, 21, 28, 60]. Due to the symmetry of nanofilm electrode in Fig. 1b, the constitutive equations for linear elastic nanofilm are 
written as follows:

$$
\sigma_{i i}=\frac{E}{(1+v)(1-2 v)}\left[(1-2 v) \varepsilon_{i i}+v \sum_{i=1}^{3} \varepsilon_{i i}\right]-\frac{E \Omega C(z, t)}{3(1-2 v)}
$$

where $\sigma_{11}, \sigma_{22}$, and $\sigma_{33}$, and $\varepsilon_{11}, \varepsilon_{22}$, and $\varepsilon_{33}$ mean the stress and strain components along $\mathrm{x}, \mathrm{y}$, and $\mathrm{z}$ directions, respectively. E represents the Young's modulus, $v$ is the Poisson's ratio, $\Omega$ is the partial molar volume of solute, and $\mathrm{C}$ is the molar solute concentration.

Considering the surface stress, the following expression in this plane case can be derived as

$$
\varepsilon_{x}=\varepsilon_{y}, \sigma_{z}=2 \lambda / R
$$

where $\lambda$ and $\mathrm{R}$ are the isotropic surface energy and the curvature of deformed electrode, as shown in Fig. $1 \mathrm{~b}$.

Therefore, the biaxial stresses in the thin film electrode are obtained by substituting Eq. (2) into Eq. (1)

$$
\sigma_{x}=\sigma_{y}=\frac{E \Omega}{3 h(1-v)} \int_{0}^{h} C(z, t) d z-\frac{E \Omega C(z, t)}{3(1-v)}-\sigma_{\text {surface }}
$$

where $\mathrm{E}$ is Young's modulus, $\Omega$ is partial molar volume of the solute, and $v$ is Poisson's ratio. $\sigma_{\text {surface }}=2 \gamma / z+\lambda / R$ [61], where $\gamma$ is surface energy. Here, the shape of Si electrode is the nanofilm with along the $\mathrm{x}$ and $\mathrm{y}$ directions, and namely the radius of $\mathrm{R}$ is infinity value. Hence, $\sigma_{\text {surface }}=2 \gamma / \mathrm{h}$ and $\sigma_{z}=0$.

Under galvanostatic operation, the solute concentration is expressed by [62]

$$
\left.C(z, t)=\frac{I h}{F D}\left\{\varphi+\frac{3(\phi-1)^{2}-1}{6}-\frac{2}{\pi^{2}} \sum_{k=1}^{\infty} \frac{(-1)^{k}}{k^{2}} \cos k \pi(\phi-1)\right] \exp \left(-k^{2} \pi^{2} \varphi\right)\right\}
$$

where $\varphi=z / h$ and $\phi=D t / h^{2} . I$ is the surface current density, D is the diffusion coefficient of the solute, and $\mathrm{F}$ is the Faraday's constant.

By substituting equation (4) into equation (3), the biaxial stresses under galvanostatic operation can be obtained as

$$
\begin{aligned}
\sigma_{x}=\sigma_{y}= & \frac{E \Omega I h}{3 F D(1-v)}\left\{\frac{2}{\pi^{2}} \sum_{k=1}^{\infty} \frac{(-1)^{k}}{k^{2}} \cos [k \pi(\phi-1)] \exp \left(-k^{2} \pi^{2} \varphi\right)-\frac{3(\phi-1)^{2}-1}{6}-\varphi\right\} \\
& +\frac{E \Omega I h}{3 F D(1-v)}\left[\varphi+\frac{2}{\pi^{3}} \sum_{k=1}^{\infty} \frac{(-1)^{k}}{k^{3}} \sin (-k \pi) \exp \left(-k^{2} \pi^{2} \varphi\right)\right]-\frac{2 \gamma}{h}
\end{aligned}
$$

Under potentiostatic operation, the solute concentration is expressed by [62]

$$
C(z, t)=C_{0}\left\{1-\frac{4}{\pi} \sum_{k=0}^{\infty} \frac{(-1)^{k}}{2 k+1} \cos \frac{\pi(\phi-1)(2 k+1)}{2} \exp \left[-\frac{\varphi \pi^{2}(2 k+1)^{2}}{4}\right]\right\}
$$


where $\varphi=z / h$ and $\phi=D t / h^{2}$.

By substituting equation (6) into equation (3), the biaxial stresses under potentiostatic operation can be derived as

$$
\begin{aligned}
\sigma_{x}=\sigma_{y}= & \frac{C_{0} E \Omega}{3(1-v)}\left\{\frac{8}{\pi^{2}} \sum_{k=0}^{\infty} \frac{(-1)^{k}}{(2 k+1)^{2}} \sin \frac{-\pi(2 k+1)}{2} \exp \left[-\frac{\varphi \pi^{2}(2 k+1)^{2}}{4}\right]\right\} \\
& +\frac{C_{0} E \Omega}{3(1-v)}\left\{\frac{4}{\pi} \sum_{k=0}^{\infty} \frac{(-1)^{k}}{2 k+1} \cos \frac{\pi(\phi-1)(2 k+1)}{2} \exp \left[-\frac{\varphi \pi^{2}(2 k+1)^{2}}{4}\right]\right\}-\frac{2 \gamma}{h}
\end{aligned}
$$

Fig. 20 shows the variations of normalized solute concentration during charging process under galvanostatic and potentiostatic operation. It is noted in Fig. $20 \mathrm{a}$ and $\mathrm{b}$ that the solute concentration increases with the increasing of time, and it continuously goes down from the upper surface to the lower surface in Si electrode. Here, the normalized embedded depth $h_{*}$ is defined as the ration of the distance away from the upper surface to the thickness of nanofim. The asymptotic behavior at an intermediate normalized embedded depth shows a sharp decrease in concentration-time cure, while the asymptotic behavior at a smaller or larger depth approaches a constant value. The slow-fast transition in concentration takes place at the relative narrow region of normalized embedded depth, which strongly relies on the embedded depth and time. At constant embedded depth, the evolution of solute concentration during galvanostatic process is more sensitive to time in comparison with potentiostatic process (see Fig. 20 c, d). This result is consistent with the result of spherical and cylindrical Si electrode in Li-ion battery [19-21].

Using Eqs. (3) and (7), the dimensionless biaxial stresses $\sigma_{*}=\sigma_{x} / \frac{E \Omega / h}{3 F D(1-v)}=\sigma_{y} / \frac{E \Omega / h}{3 F D(1-v)} \quad$ and $\sigma_{*}=\sigma_{x} / \frac{C_{0} E \Omega}{3(1-v)}=\sigma_{y} / \frac{C_{0} E \Omega}{3(1-v)}$ can be obtained during galvanostatic and potentiostatic operations (see Fig. 21). Table 1 summarizes the computational parameters $[16,19,21,63]$ used in investigating the diffusion-induced stress. The biaxial stress at the galvanostatic stage is always compressive during Li-ion insertion. However, at potentiostatic stage, the upper layer is compressive and the lower layer is tensile. With increasing the time, the compressive stage is aggravated in the upper layer, and that is released in the lower layer (Fig. 21a). It is owing to the gradient descent of solute concentration to reduce the strain energy as compared to the initial lithiation stage. This result is proved in Fig. 8, where the low strain is found in the upper layer. However, during potentiostatic stage, the opposite phenomenon is observed in Fig. 21b. The charging stage significantly affects the stress distribution, which directly determines the crack nucleation and further controls the Li-ion battery life. When the embedded depth is constant, the serious stress fluctuation occurs only in the relative 
short time, such as about 0.2 at galvanostatic operation and about 0.4 at potentiostatic operation. In other word, the galvanostatic charging has more obvious advantages to keep stable stress stage than the potentiostatic charging. The biaxial stresses during Li-ion extraction have the same magnitude of that during Li-ion insertion, while they have the opposite sign.

\section{Conclusion}

In this paper, the plastic deformation and structural stability of lithiated Si anode are investigated and analyzed by MD simulations. It is found to be related upon the effects of crystal orientation, cycling number, temperature and electric field strength. We also perform the analytical expressions from linear elastic mechanics to predict the diffusion-induced stress in the equilibrated lithiated Si anode. The results of atomic simulations reveal that the different crystal orientations, cycling numbers and temperatures as well as electric field strengths would affect the volume expansion, surface topography, structural stability and nanovoid in lithiated Si electrode, and further have an influence on the capacity and life of Li-ion battery during cycling. The simultaneous coherent phase transformation from $\alpha$-Si to the $\beta$-Si is found and determined in the RDF curve after the first cycling, where the nucleation of $\beta$-Si occurs underneath lithiated Si layer in the high-strain regions. Compared with the effects of cycling number and electric field strength, the crystal orientation and temperature effects play a weak role in the plastic deformation and structural stability of Si electrode during cycling, resulting in the formation of nanovoids. In addition, the surface topography, and the size and number of nanovoid are dependent upon the cycling number and temperature as well as electric field strength. Theoretical model predicts that the galvanostatic charging has more obvious advantages to keep structural stability than the potentiostatic charging. Considering the coupling effects of crystal orientation, cycling number, temperature and electric field strength to dominate the structural stability and damage degree in Si anode, the current results could optimize the appropriate parameters for prolonging Li-ion battery life.

\section{Acknowledgements}

The authors would like to deeply appreciate the support from the NNSFC (11572118 and 11772122), the

Hunan Provincial Science Fund for Distinguished Young Scholars (2015JJ1006), the Project of Innovation-driven Plan in Central South University of China (2015CX004), the State Key Laboratory of Powder Metallurgy, and the National Key Research and Development Program of China (2016YFB0700300).

\section{References}

[1] C.Y. Wang, G. Zhang, S. Ge, T. Xu, Y. Ji, X.G. Yang, Y. Leng, Lithium-ion battery structure that self-heats 
at low temperatures, Nature 529 (2016) 515-518.

[2] A.M. Korsunsky, T. Sui, B. Song, Explicit formulae for the internal stress in spherical particles of active material within lithium ion battery cathodes during charging and discharging, Mater. Design 69 (2015) 247-252.

[3] K.K. Lee, K. Park, H. Lee, Y. Noh, D. Kossowska, K. Kwak, M. Cho, Ultrafast fluxional exchange dynamics in electrolyte solvation sheath of lithium ion battery, Nat. Commun. 8 (2017) 14658-14666.

[4] J. Xu, L. Wang, J. Guan, S. Yin, Coupled effect of strain rate and solvent on dynamic mechanical behaviors of separators in lithium ion batteries, Mater. Design 95 (2016) 319-328.

[5] B. Ding, H. Wu, Z. Xu, X. Li, H. Gao, Stress effects on lithiation in silicon, Nano Energy 38 (2017) 486-493.

[6] A.O. Kondrakov, A. Schmidt, J. Xu, H. Geßwein, R. Mönig, P. Hartmann, J. Janek, Anisotropic lattice strain and mechanical degradation of high-and low-nickel ncm cathode materials for li-ion batteries, J. Phys. Chem. C 121 (2017) 3286-3294.

[7] B. Ding, X. Li, X. Zhang, H. Wu, Z. Xu, H. Gao, Brittle versus ductile fracture mechanism transition in amorphous lithiated silicon: From intrinsic nanoscale cavitation to shear banding, Nano Energy 18 (2015) 89-96.

[8] S. Hao, B. Zhang, S. Ball, B. Hu, J. Wu, Y. Huang, Porous and hollow NiO microspheres for high capacity and long-life anode materials of Li-ion batteries, Mater. Design 92 (2016) 160-165.

[9] X. Hu, S. Kobayashi, Y.H. Ikuhara, C.A. Fisher, Y. Fujiwara, K. Hoshikawa, Y. Ikuhara, Atomic scale imaging of structural variations in $\mathrm{La}_{(1-\mathrm{x}) / 3} \mathrm{Li}_{\mathrm{x}} \mathrm{NbO}_{3}(0 \leq \mathrm{x} \leq 0.13)$ solid electrolytes, Acta Mater. 123 (2017) 167-176.

[10] Y. Sun, N. Liu, Y. Cui, Promises and challenges of nanomaterials for lithium-based rechargeable batteries, Nat. Energy 1 (2016) 16071-16082.

[11] A. Ramasubramanian, V. Yurkiv, A. Najafi, A. Khounsary, R. Shahbazian-Yassar, F. Mashayek, A comparative study on continuum-scale modeling of elasto-plastic deformation in rechargeable ion batteries, J. Electrochem. Soc. 164 (2017) 3418-3425.

[12] F. Shi, Z. Song, P.N. Ross, G.A. Somorjai, R.O. Ritchie, K. Komvopoulos, Failure mechanisms of single-crystal silicon electrodes in lithium-ion batteries, Nat. Commun. 7 (2016) 11886-11894.

[13] G. Bucci, Y.M. Chiang, W.C. Carter, Formulation of the coupled electrochemical-mechanical boundary-value problem, with applications to transport of multiple charged species, Acta Mater. 104 (2016) 
$33-51$.

[14] K. Zhao, L. Zhang, R. Xia, Y. Dong, W. Xu, C. Niu, L. Mai, $\mathrm{SnO}_{2}$ quantum dots@graphene oxide as a high-rate and long-life anode material for lithium-ion batteries, Small 12 (2016) 588-594.

[15] M. Freire, N.V. Kosova, C. Jordy, D. Chateigner, O.I. Lebedev, A. Maignan, V. Pralong, A new active LiMnO compound for high energy density Li-ion batteries, Nat. Mater. 15 (2016) 173-177.

[16] J. Li, D. Lu, Q. Fang, Y. Liu, P. Wen, Cooperative surface effect and dislocation effect in lithium ion battery electrode, Solid State Ionics 274 (2015) 46-54.

[17] S.M. Khosrownejad, W.A. Curtin, Crack growth and fracture toughness of amorphous Li-Si anodes: Mechanisms and role of charging/discharging studied by atomistic simulations, J. Mech. Phys. Solids 107 (2017) 542-559.

[18] R. Deshpande, Y.T. Cheng, M.W. Verbrugge, Modeling diffusion-induced stress in nanowire electrode structures, J. Power Sources 195 (2010) 5081-5088.

[19] J. Li, Q. Fang, F. Liu, Y. Liu, Analytical modeling of dislocation effect on diffusion induced stress in a cylindrical lithium ion battery electrode, J. Power Sources 272 (2014) 121-127.

[20] D. Li, Y. Wang, J. Hu, B. Lu, Y.T. Cheng, J. Zhang, In situ measurement of mechanical property and stress evolution in a composite silicon electrode, J. Power Sources 366 (2017) 80-85.

[21] B. Chen, J. Zhou, R. Cai, Analytical model for crack propagation in spherical nano electrodes of lithium-ion batteries, Electrochim. Acta 210 (2016) 7-14.

[22] Y. Yao, M.T. McDowell, I. Ryu, H. Wu, N. Liu, L. Hu, Y. Cui, Interconnected silicon hollow nanospheres for lithium-ion battery anodes with long cycle life, Nano Lett. 11 (2011) 2949-2954.

[23] K. Zhao, G.A. Tritsaris, M. Pharr, W.L. Wang, O. Okeke, Z. Suo, J.J. Vlassak, E. Kaxiras, Reactive flow in silicon electrodes assisted by the insertion of lithium, Nano Lett. 12 (2012) 4397-4403.

[24] A. Ostadhossein, E.D. Cubuk, G.A. Tritsaris, E. Kaxiras, S.L. Zhang, A.C.T. van Duin, Stress effects on the initial lithiation of crystalline silicon nanowires: reactive molecular dynamics simulations using ReaxFF, Phys. Chem. Chem. Phys. 17 (2015) 3832-3840.

[25] Z. Cui, F. Gao, Z. Cui, J. Qu, A second nearest-neighbor embedded atom method interatomic potential for Li-Si alloys, J. Power Sources 207 (2012) 150-159.

[26] J. Stadler, R. Mikulla, H.R. Trebin, IMD: a software package for molecular dynamics studies on parallel computers, Int. J. Mod. Phys. C 8 (1997) 1131-1140.

[27] H. Haftbaradaran, J. Song, W. A. Curtin, H. Gao, Continuum and atomistic models of strongly coupled 
diffusion, stress, and solute concentration. J. Power Sources 196 (2011) 361-370.

[28] J. Li, Q.H. Fang, H. Wu, Y. Liu, P. Wen, Investigation into diffusion induced plastic deformation behavior in hollow lithium ion battery electrode revealed by analytical model and atomistic simulation, Electrochim. Acta 178 (2015) 597-607.

[29] M. Thompson, L.E. Cheran, M. Zhang, M. Chacko, H. Huo, S. Sadeghi, Label-free detection of nucleic acid and protein microarrays by scanning Kelvin nanoprobe, Bios. Bioelectron. 20 (2005) 1471-1481.

[30] I.V. Thorat, D.E. Stephenson, N.A. Zacharias, K. Zaghib, J.N. Harb, D.R. Wheeler, Quantifying tortuosity in porous Li-ion battery materials, J. Power Sources 188 (2009) 592-600.

[31] P.K. Cong, P. Fellner, Influence of the pretreatment of silicon powder by electroless nickel coating on its codeposition with nickel, Chem. Pap. 48 (1994) 326-329.

[32] A. Stukowski, Visualization and analysis of atomistic simulation data with OVITO-the Open Visualization Tool, Model. Simu. Mater. Sci. Eng. 18 (2009) 015012-015019.

[33] N. Balke, S. Jesse, A.N. Morozovska, E. Eliseev, D.W. Chung, Y. Kim, S.V. Kalinin, Nanoscale mapping of ion diffusion in a lithium-ion battery cathode, Nat. Nanotechnol. 5 (2010) 749-754.

[34] A. Kuhn, P. Sreeraj, R. Poettgen, H.D. Wiemhoefer, M. Wilkening, P. Heitjans, Li ion diffusion in the anode material $\mathrm{Li}_{12} \mathrm{Si}_{7}$ : Ultrafast quasi-1D diffusion and two distinct fast 3D jump processes separately revealed by ${ }^{7} \mathrm{Li}$ NMR relaxometry, J. Am. Chem. Soc. 133 (2011) 11018-11021.

[35] S.I. Nishimura, G. Kobayashi, K. Ohoyama, R. Kanno, M. Yashima, A. Yamada, Experimental visualization of lithium diffusion in $\mathrm{Li}_{x} \mathrm{FePO}_{4}$, Nat. Mater. 7 (2008) 707-711.

[36] Q. Zhang, W. Zhang, W. Wan, Y. Cui, E. Wang, Lithium insertion in silicon nanowires: an ab initio study, Nano Lett. 10 (2010) 3243-3249.

[37] O.I. Malyi, T.L. Tan, S. Manzhos, A comparative computational study of structures, diffusion, and dopant interactions between Li and Na insertion into Si, Appl. Phys. Exp. 6 (2013) 027301-027303.

[38] P. Johari, Y. Qi, V.B. Shenoy, The mixing mechanism during lithiation of Si negative electrode in Li-ion batteries: an ab initio molecular dynamics study, Nano Lett. 11 (2011) 5494-5500.

[39] S.P. Kim, A.C. Van Duin, V.B. Shenoy, Effect of electrolytes on the structure and evolution of the solid electrolyte interphase (SEI) in Li-ion batteries: A molecular dynamics study, J. Power Sources 196 (2011) 8590-8597.

[40] E. Peled, The electrochemical behavior of alkali and alkaline earth metals in nonaqueous battery systems-the solid electrolyte interphase model, J. Electrochem. Soc. 126 (1979) 2047-2051. 
[41] D.E. Kim, S.I. Oh, Atomistic simulation of structural phase transformations in monocrystalline silicon induced by nanoindentation, Nanotechnology 17 (2006) 2259-2265.

[42] Y. Hong, N. Zhang, M.A. Zaeem, Metastable phase transformation and deformation twinning induced hardening-stiffening mechanism in compression of silicon nanoparticles, Acta Mater. 145 (2018) 8-18.

[43] J.J. Fan, J. Li, Z. Huang, P.H. Wen, C.G. Bailey, Grain size effects on indentation-induced plastic deformation and amorphization process of polycrystalline silicon, Comp. Mater. Sci. 144 (2018) 113-119.

[44] R. Grantab, V.B. Shenoy, Location-and orientation-dependent progressive crack propagation in cylindrical graphite electrode particles, J. Electrochem. Soc. 158 (2011) 948-954.

[45] J. Vetter, P. Novák, M.R. Wagner, C. Veit, K.C. Möller, J.O. Besenhard, M. Winter, M. Wohlfahrt-Mehrens, C. Vogler, A. Hammoushe, Ageing mechanisms in lithium-ion batteries, J. Power Sources 147 (2005) 269-281.

[46] A. Verma, K. Smith, S. Santhanagopalan, D. Abraham, K.P. Yao, P.P. Mukherjee, Galvanostatic intermittent titration and performance based analysis of $\mathrm{LiNi}_{0.5} \mathrm{Co}_{0.2} \mathrm{Mn}_{0.3} \mathrm{O}_{2}$ cathode, J. Electrochem. Soc. 164 (2017) 3380-3392.

[47] K.A. Jarvis, C.C. Wang, J.C. Knight, L. Rabenberg, A. Manthiram, P.J. Ferreira,. Formation and effect of orientation domains in layered oxide cathodes of lithium-ion batteries, Acta Mater. 108 (2016) 264-270.

[48] J.C. Jamieson, Crystal structures at high pressures of metallic modifications of silicon and germanium, Science 139 (1963) 762-764.

[49] C.M. Wang, X. Li, Z. Wang, W. Xu, J. Liu, F. Gao, Z. Liu, In situ TEM investigation of congruent phase transition and structural evolution of nanostructured silicon/carbon anode for lithium ion batteries, Nano Lett. 12 (2012) 1624-1632.

[50] M.E. Garcia, S.H. Garofalini, Molecular dynamics simulation of the effect of crystal orientation on lithium-ion diffusion at the $\mathrm{V}_{2} \mathrm{O}_{5} / \mathrm{Li}_{2} \mathrm{SiO}_{3}$ interface, J. Electrochem. Soc. 146 (1999) 840-849.

[51] B. Chen, S. Chu, R. Cai, J. Zhou, The effect of diffusion induced fatigue stress on capacity loss in nano silicon particle electrodes during cycling, J. Electrochem. Soc. 163 (2016) 2592-2599.

[52] W. Liang, L. Hong, H. Yang, F. Fan, Y. Liu, H. Li, J. Li, J.Y. Huang, L.Q. Chen, T. Zhu, S. Zhang, Nanovoid formation and annihilation in gallium nanodroplets under lithiation-delithiation cycling, Nano Lett. 13 (2013) 5212-5217.

[53] Y.S. Jung, P. Lu, A.S. Cavanagh, C. Ban, G.H. Kim, S.H. Lee, S.M. George, S.J. Harris, A.C. Dillon, Unexpected improved performance of ALD coated $\mathrm{LiCoO}_{2} / \mathrm{Graphite} \mathrm{Li}$-ion batteries, Adv. Energy Mater. 3 
(2013) 213-219.

[54] B. Lu, Y. Song, Q. Zhang, J. Pan, Y.T. Cheng, J. Zhang, Voltage hysteresis of lithium ion batteries caused by mechanical stress, Phys. Chem. Chem. Phys. 18 (2016) 4721-4727.

[55] S. Takai, M. Kamata, S. Fujine, K. Yoneda, K. Kanda, T. Esaka, Diffusion coefficient measurement of lithium ion in sintered $\mathrm{Li}_{1.33} \mathrm{Ti}_{1.67} \mathrm{O}_{4}$ by means of neutron radiography, Solid State Ionics 123 (1999) 165-172.

[56] Y. Xiao, F.C. Zhang, J.I. Han, Electrical structures, magnetic polaron and lithium ion dynamics in three transition metal doped $\mathrm{LiFe}_{1-x} \mathrm{M}_{x} \mathrm{PO}_{4}(\mathrm{M}=\mathrm{Mn}, \mathrm{Co}$ and $\mathrm{La})$ cathode material for $\mathrm{Li}$ ion batteries from density functional theory study, Solid State Ionics 294 (2016) 73-81.

[57] J. Shi, Z. Wang, Y.Q. Fu, Atomistic study of lithium ion dynamics in $\mathrm{Li}_{12} \mathrm{Si}_{7}$, Electrochim. Acta 186 (2015) $71-75$.

[58] B. Key, R. Bhattacharyya, M. Morcrette, V. Seznec, J.M. Tarascon, C.P. Grey, Real-time NMR investigations of structural changes in silicon electrodes for lithium-ion batteries, J. Am. Chem. Soc. 131 (2009) 9239-9249.

[59] B. Key, M. Morcrette, J.M. Tarascon, C.P. Grey, Pair distribution function analysis and solid state NMR studies of silicon electrodes for lithium ion batteries: understanding the (de)lithiation mechanisms, J. Am. Chem. Soc. 133 (2010) 503-512.

[60] X. Hu, Y. Zhao, R. Cai, J. Zhou, Surface effected fracture behavior of nano-spherical electrodes during lithiation reaction, Mater. Sci. Eng. A 707 (2017) 92-100.

[61] R.C. Cammarata, T.M. Trimble, D.J. Srolovitz, Surface stress model for intrinsic stresses in thin films, J. Mater. Res. 15 (2000) 2468-2474.

[62] J. Crank, The mathematics of diffusion, Oxford University Press, Oxford, 1980.

[63] T.K. Bhandakkar, H. Gao, Cohesive modeling of crack nucleation under diffusion induced stresses in a thin strip: implications on the critical size for flaw tolerant battery electrodes, Int. J. Solids Struct. 47 (2010) 1424-1434. 
Table 1 Material properties and constants used in Si electrode.

\begin{tabular}{lc}
\hline Name & Value \\
\hline Yong's modulus of Li-Si, $E$ & $30-80 \mathrm{Gpa}$ \\
Poisson's ratio, $v$ & 0.22 \\
Diffusion coefficient, $D$ & $1.2 \times 10^{-18} \mathrm{~m}^{2} \mathrm{~s}^{-1}$ \\
Initial solute concentration, $\mathrm{C}_{0}$ & $1 \times 10^{4} \mathrm{~mol} \mathrm{~m}^{-3}$ \\
Partial molar volume of solute, $\Omega$ & $2 \times 10^{-5} \mathrm{~m}^{3} \mathrm{~mol}^{-1}$ \\
Thickness of nanofilm electrode, $\mathrm{h}$ & $2 \times 10^{-8} \mathrm{~m}$ \\
Surface energy, $\gamma$ & $1 \mathrm{~J} \mathrm{~m}^{-2}$ \\
Surface current density, I & $0.011 \mathrm{~A} \mathrm{~m}^{-2}$ \\
Faraday's constant, F & $96486.7 \mathrm{C} \mathrm{mol}^{-1}$ \\
\hline
\end{tabular}

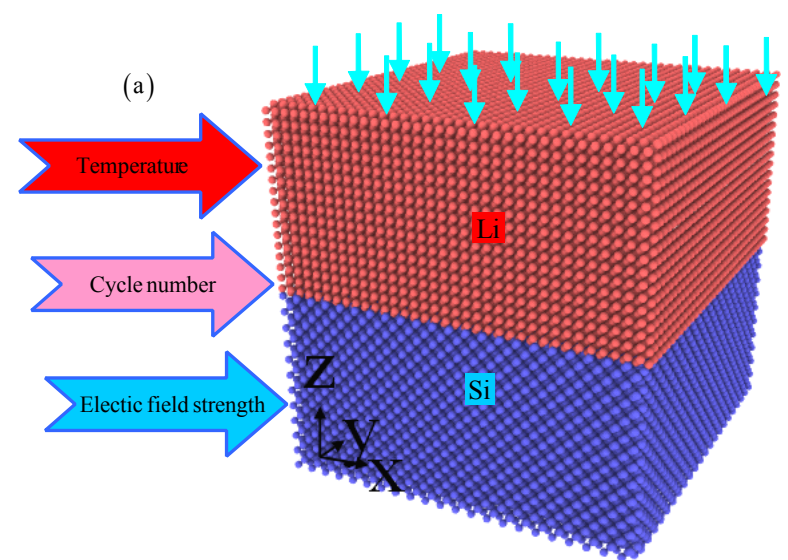

(b)

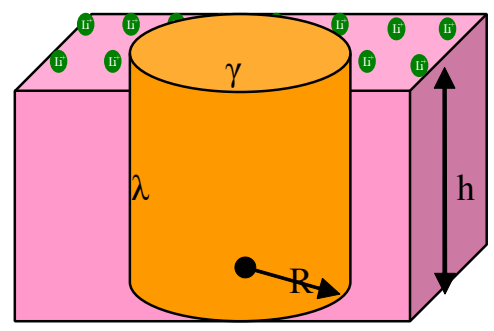

Fig. 1. Three-dimensional MD model of lithiation and delithiation in Li and Si electrodes under electric field (a). Schematic diagram of thin film active layer with surface stresses during diffusion model (b). 
(a)

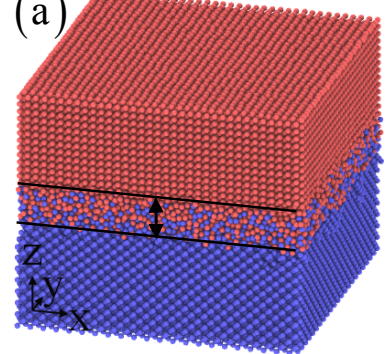

(e)

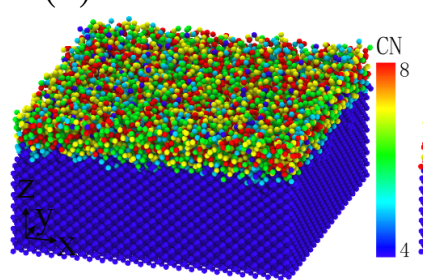

(i)

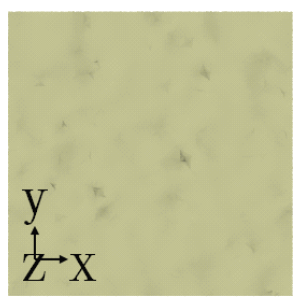

(b)

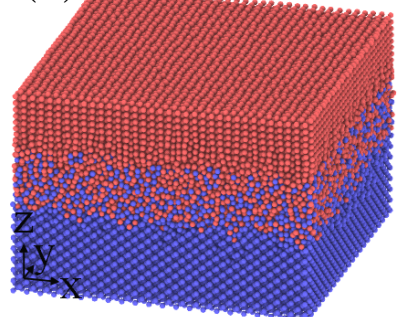

(f)

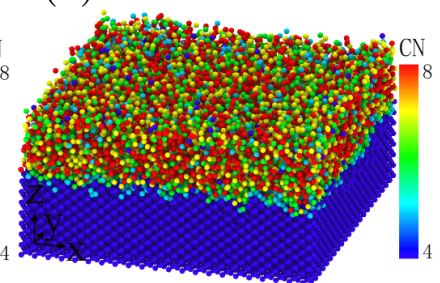

(j)

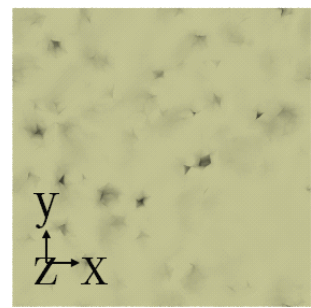

(c)

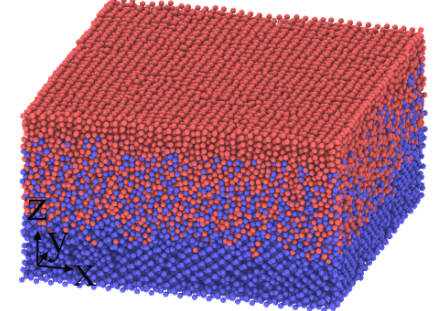

(g)

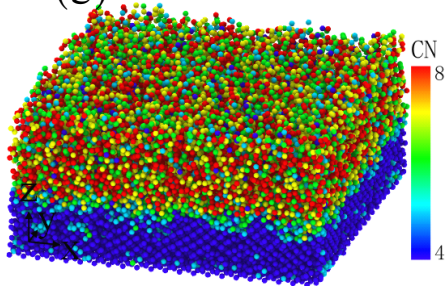

(k)

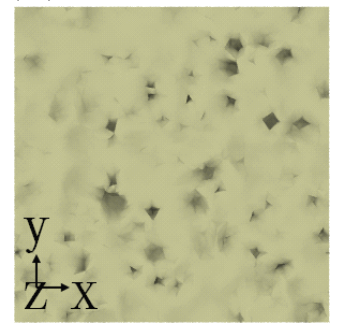

(d)

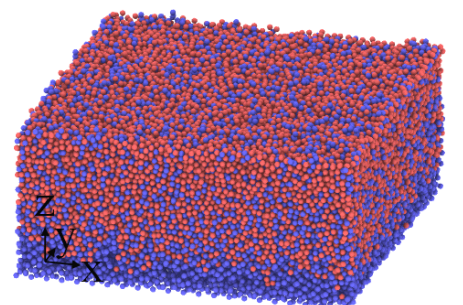

(h)

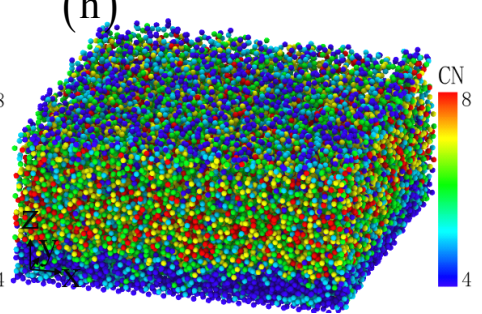

(1)

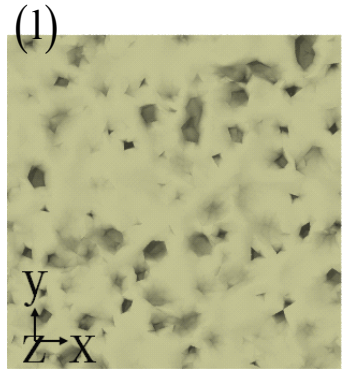

Fig. 2. The deformation behavior of Li and Si electrodes at the different lithiated depths (a-d): $1.25 \mathrm{~nm}, 2.5 \mathrm{~nm}$, $3.75 \mathrm{~nm}$, and $5 \mathrm{~nm}$. The microstructural evolution of Si anode at the different lithiated depths (e-h): $1.25 \mathrm{~nm}$, $2.5 \mathrm{~nm}, 3.75 \mathrm{~nm}$, and $5 \mathrm{~nm}$, where the atoms are colored according to the $\mathrm{CN}$. Here, $\mathrm{CN}=4$ represents $\alpha$-Si (diamond cubic structure, Si-I phase), $\mathrm{CN}=5-7$ represents $\beta$-Si (body-centred tetragonal structure, Si-II phase) and bct5, and $\mathrm{CN}=>8$ represents amorphous $\mathrm{Si}$ [41]. Li-ions are excluded for a clearer visualization of $\mathrm{Si}$ anode. The corresponding surface morphology with increase of lithiated depth (i-1), where the dark regions mean the nanovoid and and light regions mean the smooth surface. 
(a)

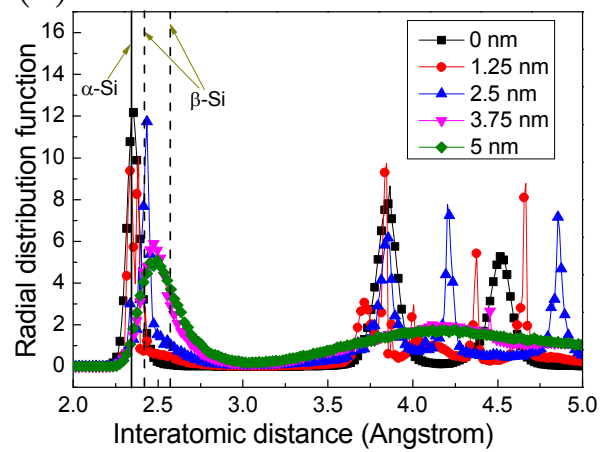

(b)

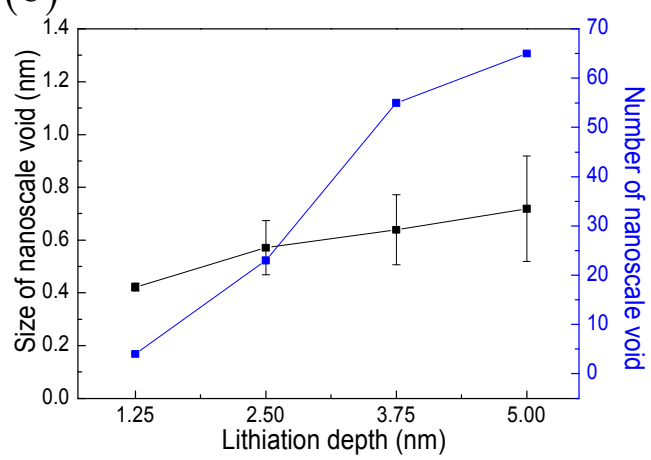

Fig. 3. The RDF showing interatomic Si-Si bond length (a), and the size and number of nanoscale void generated at surface of Si anode (b) at the different lithiated depths.

(a)
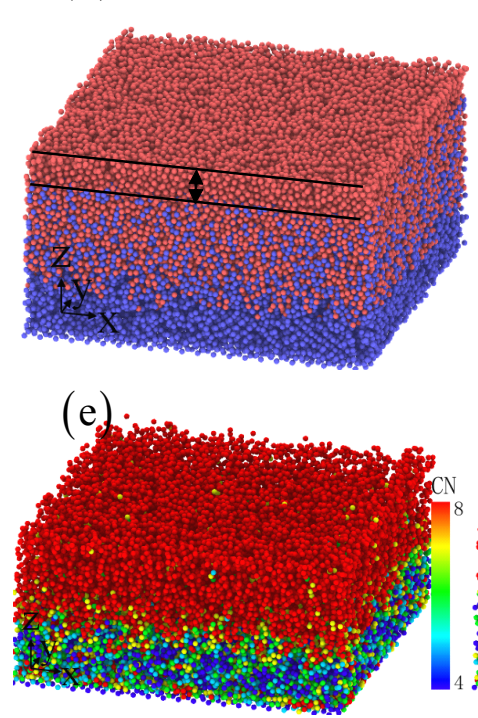

(b)
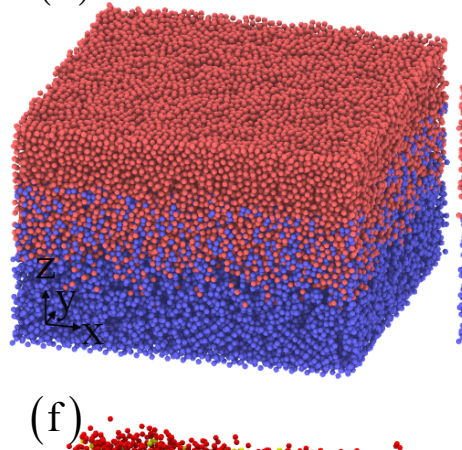

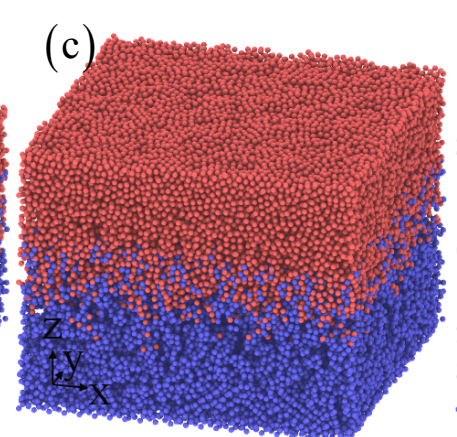

$(\mathrm{g})$

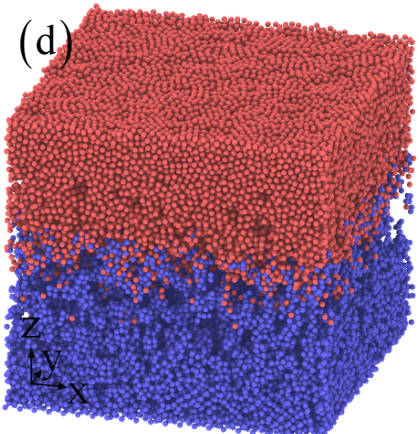

(h)

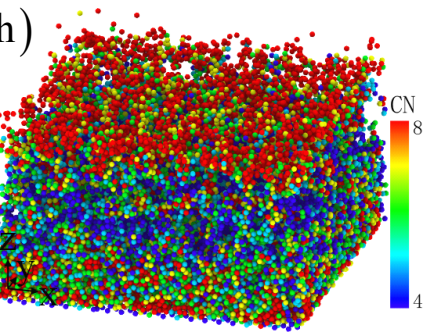

Fig. 4. The deformation behavior of $\mathrm{Li}$ and $\mathrm{Si}$ electrodes at the different delithiated depths (a-d): $1.25 \mathrm{~nm}, 2.5$ $\mathrm{nm}, 3.75 \mathrm{~nm}$, and $5 \mathrm{~nm}$. The microstructural evolution of $\mathrm{Li}$ and $\mathrm{Si}$ electrodes at the different delithiated depths (e-h): $1.25 \mathrm{~nm}, 2.5 \mathrm{~nm}, 3.75 \mathrm{~nm}$, and $5 \mathrm{~nm}$, where the atoms are colored according to the CN. 
(a)

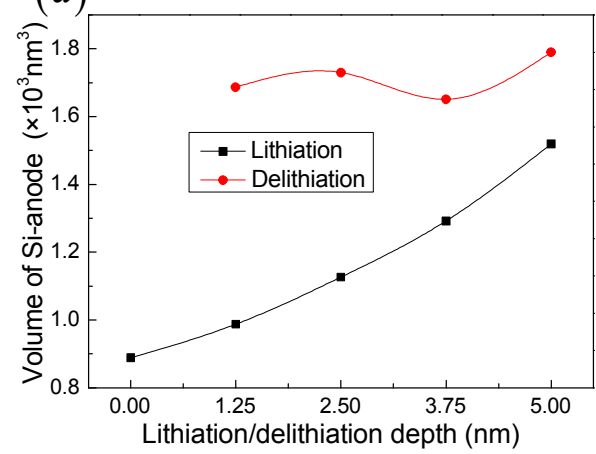

(b)

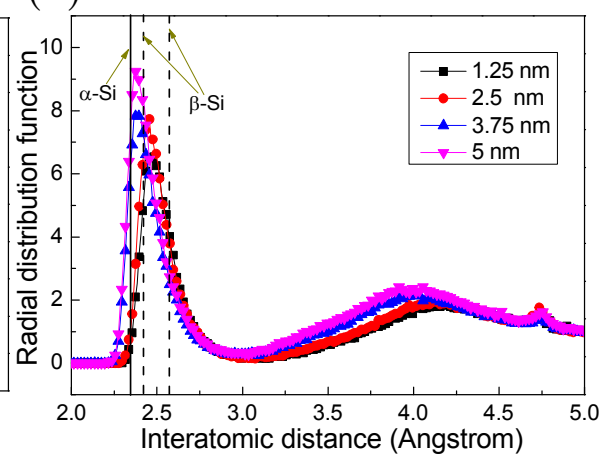

(c)

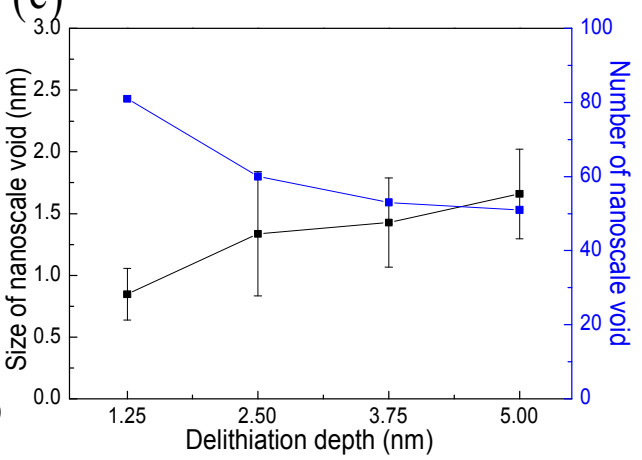

Fig. 5. The volume of Si electrode at the lithiation and delithiation depths (a), RDF showing interatomic Si-Si bond length (b), and the size and number of nanoscale void generated at surface of Si anode (c) at different delithiated depths.

(a)

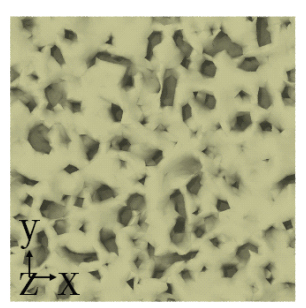

(b)

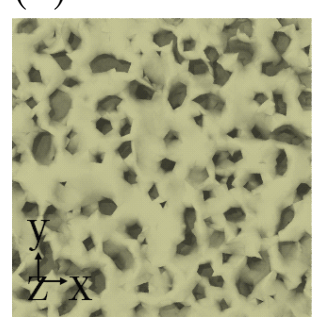

(c)

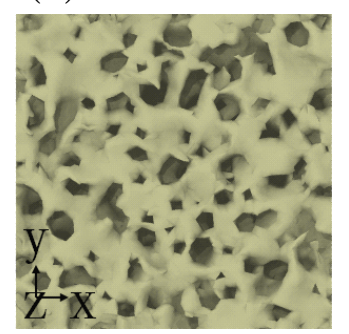

(d)

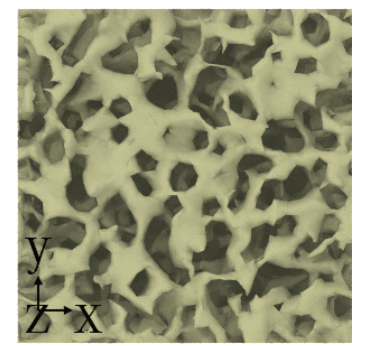

Fig. 6. The surface morphology of Si electrode at the different delithiated depths (a-d): $1.25 \mathrm{~nm}, 2.5 \mathrm{~nm}, 3.75$ $\mathrm{nm}$, and $5 \mathrm{~nm}$.

(a)

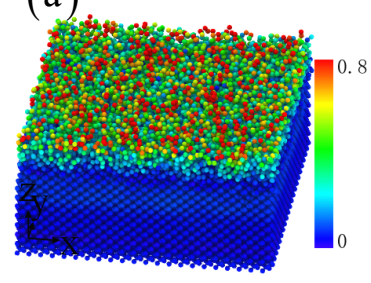

(e)

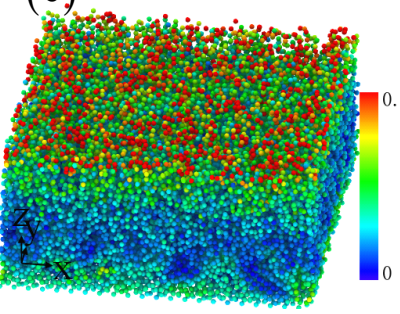

(b)

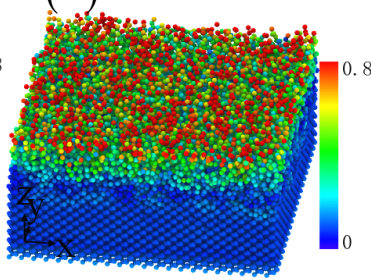

(f).

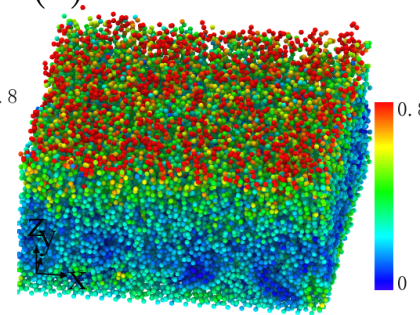

(c)

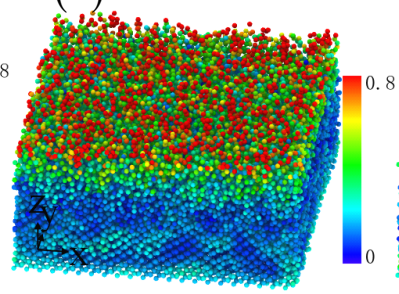

(g) (d)

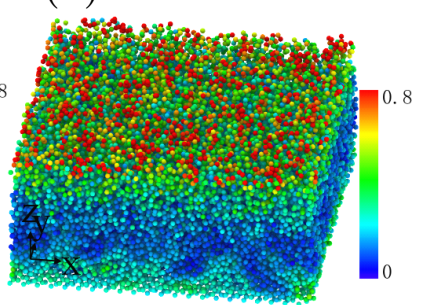

(h)
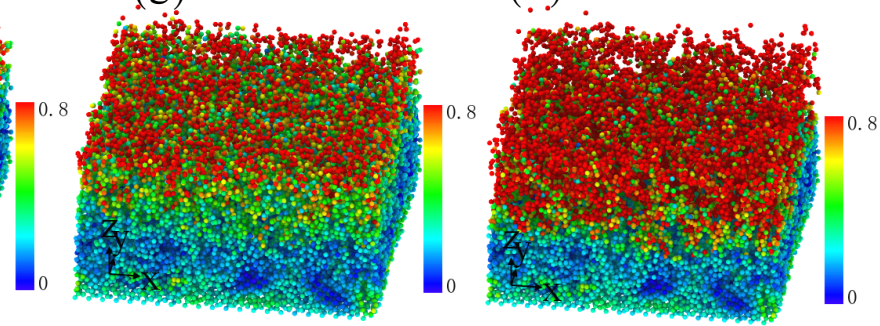

Fig. 7. The strain distribution of Si electrode at the different lithiated depths (a-d): $1.25 \mathrm{~nm}, 2.5 \mathrm{~nm}, 3.75 \mathrm{~nm}$, and $5 \mathrm{~nm}$, and at the different delithiated depths (e-h): $1.25 \mathrm{~nm}, 2.5 \mathrm{~nm}, 3.75 \mathrm{~nm}$, and $5 \mathrm{~nm}$, where atoms are colored according to the strain values. Li-ions are excluded for a clearer visualization of Si anode. 
(a)
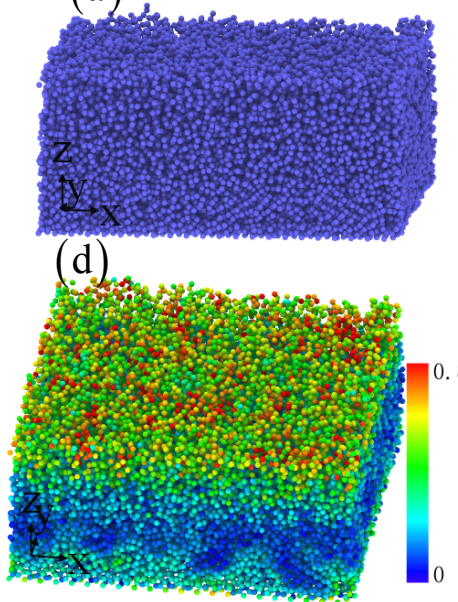

$(\mathrm{g})$

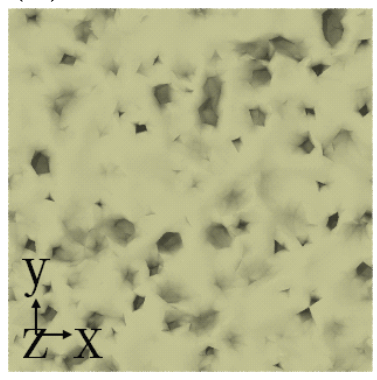

(b)

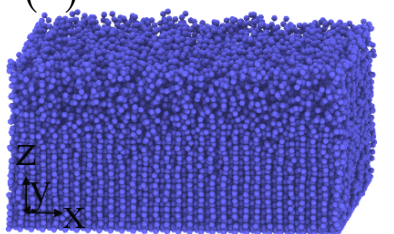

(e)

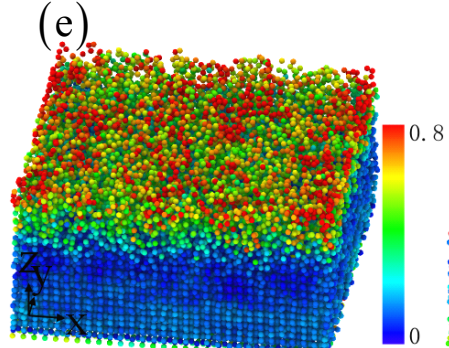

(h)

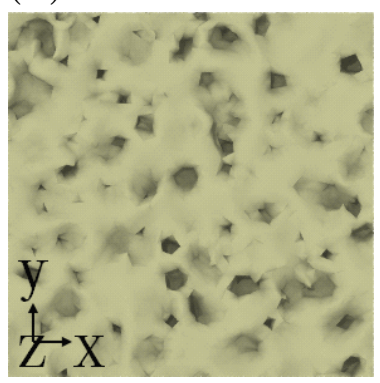

(c)
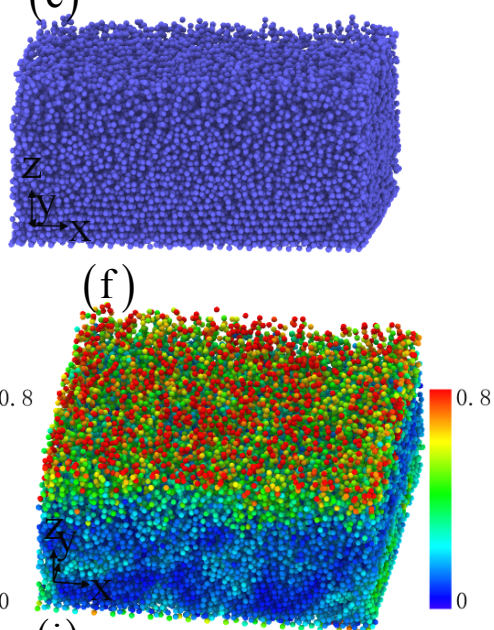

(i)

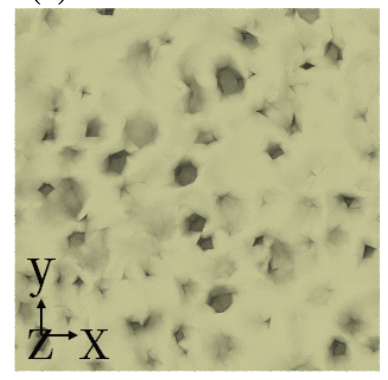

Fig. 8. The deformation behavior of Si electrode (a-c), the strain distribution of Si electrode (d-f), and the corresponding surface morphology of Si electrode (g-i) at different crystal orientations: [100] (a,d,g), [110] $(\mathrm{b}, \mathrm{e}, \mathrm{f})$, and $[111](\mathrm{c}, \mathrm{f}, \mathrm{i})$.

(a)

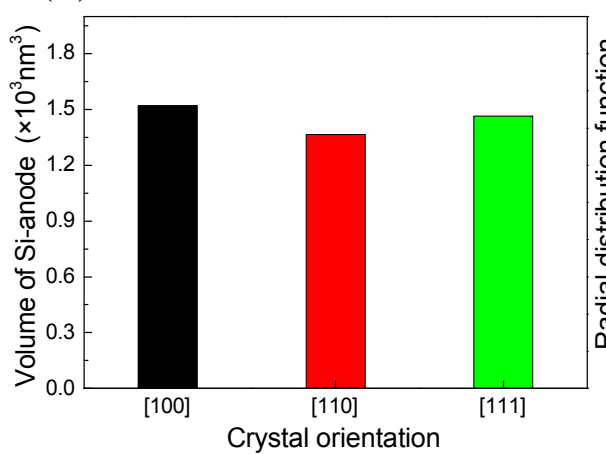

(b)

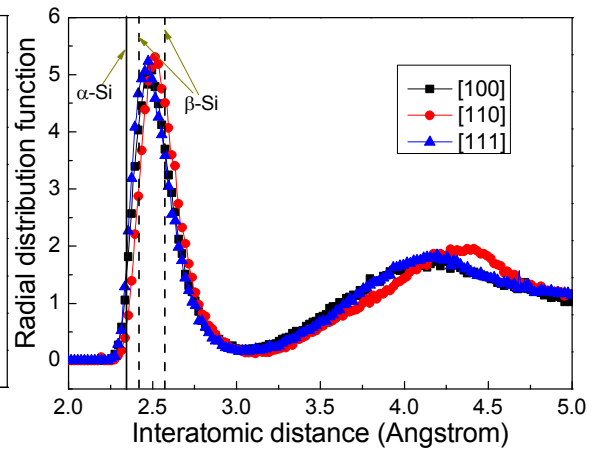

(c)

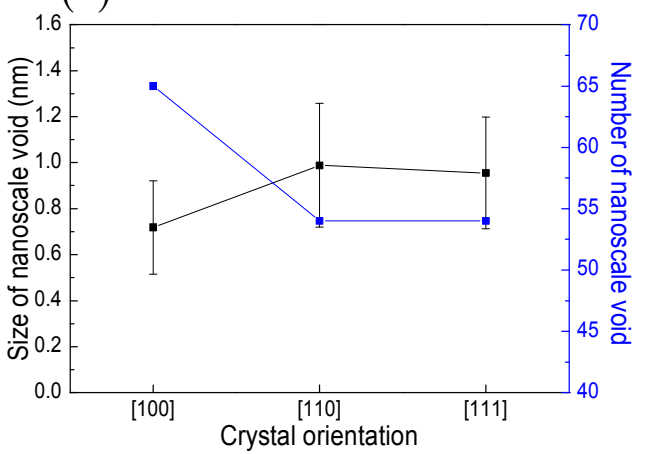

Fig. 9. The volume of Si electrode (a), RDF showing interatomic bond length (b), and the size and number of nanoscale void generated at surface of Si anode (c) at different crystal orientations. 

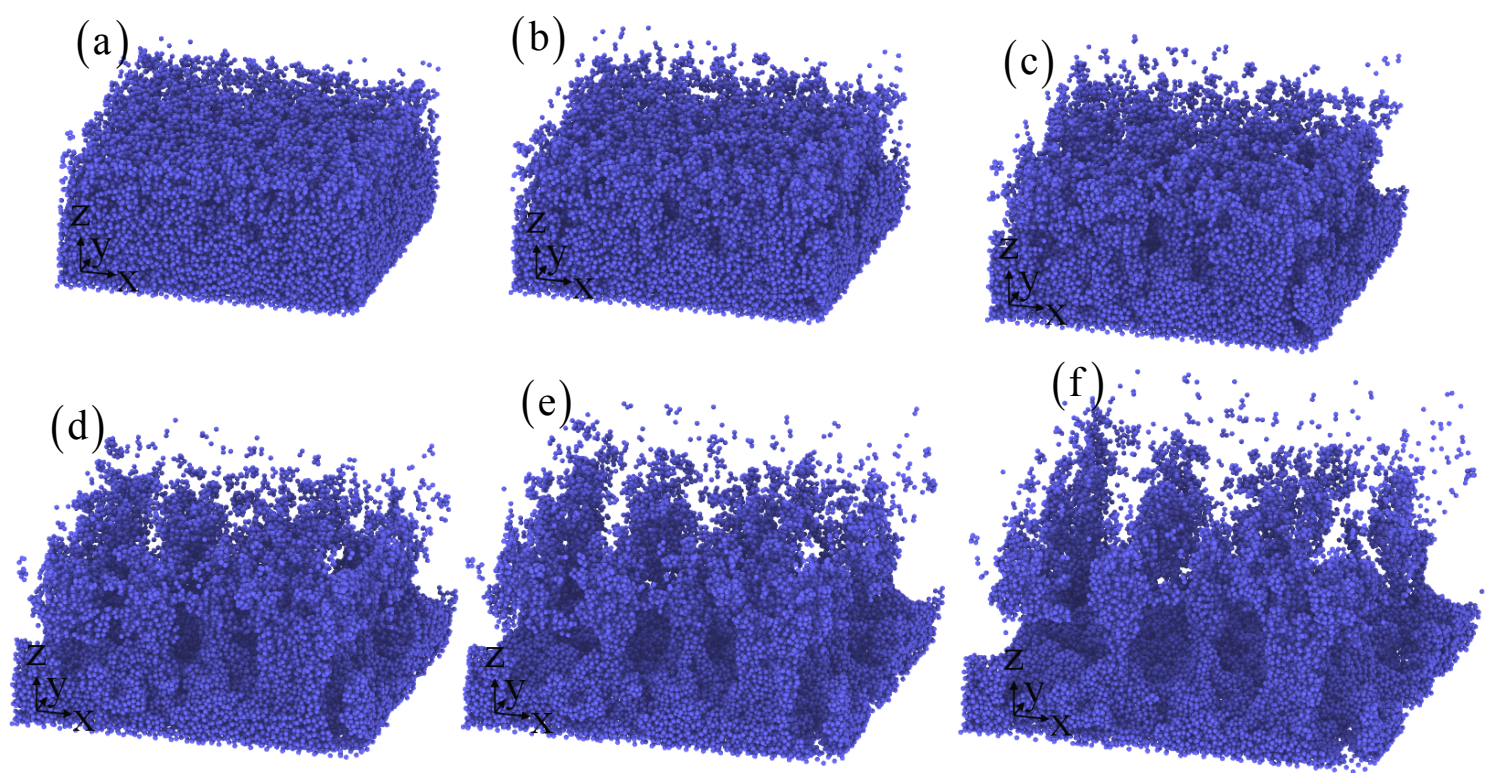

Fig. 10. The deformation behavior of Si electrode at the different cycle numbers (a-f): 2, 3, 4, 5, 6 and 7 after lithiation process.

(a)

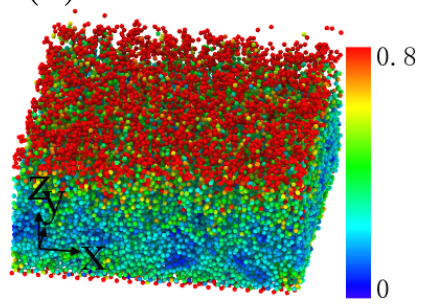

(d).

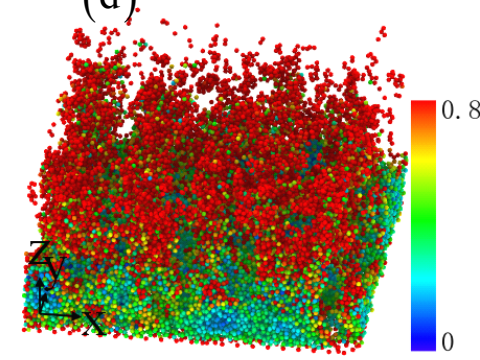

(b)

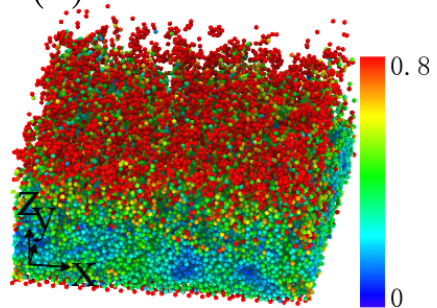

(e)

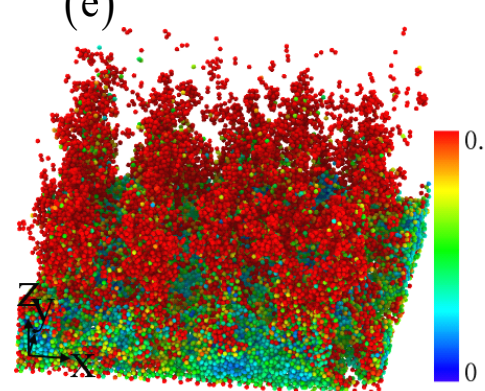

(c).

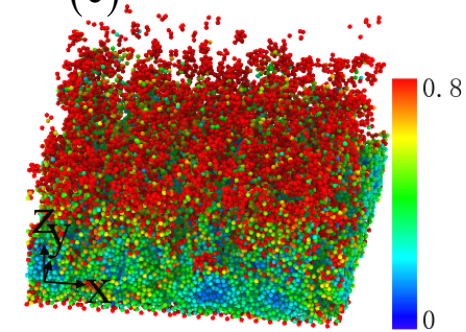

$(\mathrm{f})$

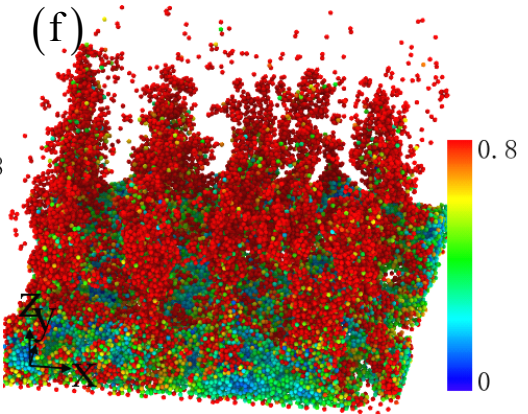

Fig. 11. The strain distribution of Si electrode at the different cycle numbers (a-f): 2, 3, 4, 5, 6 and 7, where atoms are colored according to the strain values. 
(a)

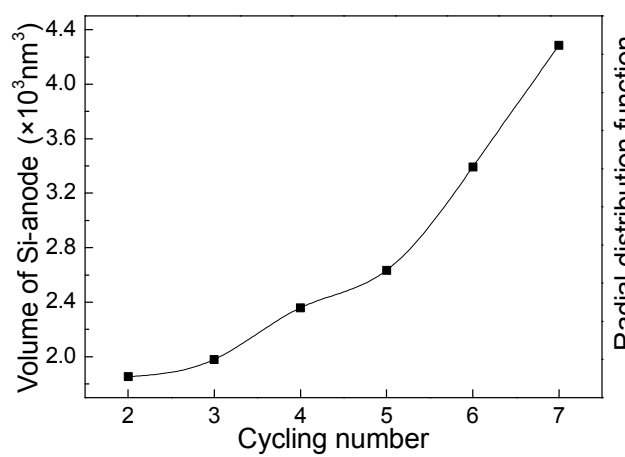

(b)

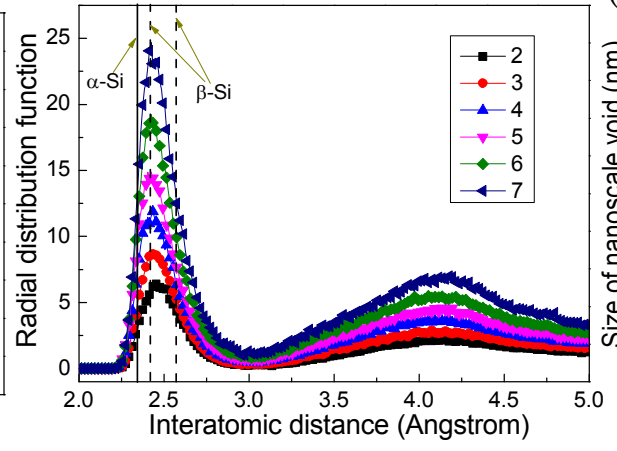

(c)

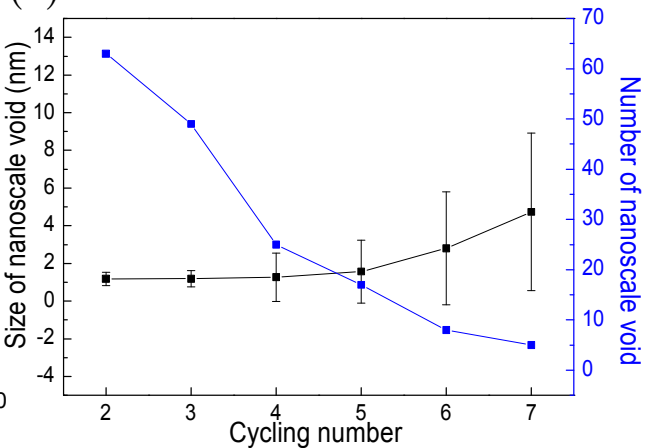

Fig. 12. The volume of Si electrode (a), RDF showing interatomic Si-Si bond length (b), and the size and number of nanoscale void generated at surface of Si anode (c) at the different cycle numbers.

(a)

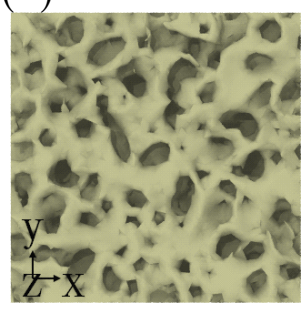

(d)

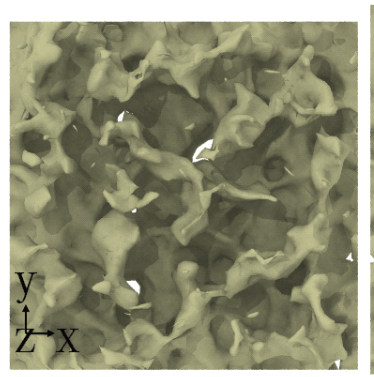

(b)

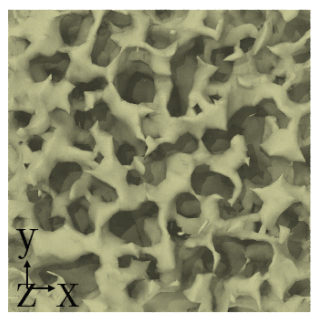

(e)

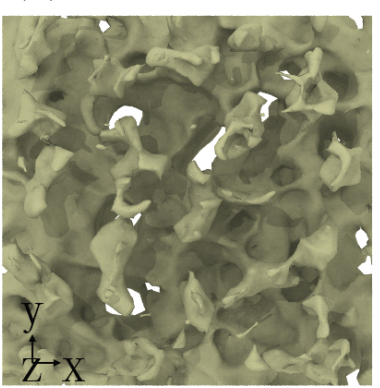

(c)

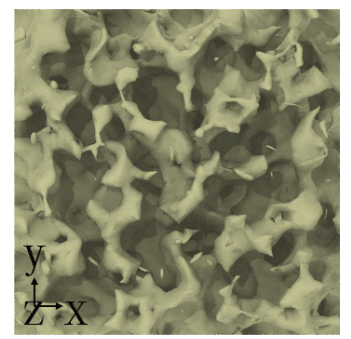

(f)

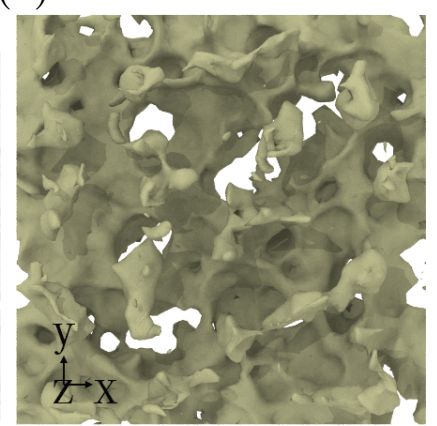

Fig. 13. The surface morphology of Si electrode at the different cycle numbers (a-f): 2, 3, 4, 5, 6 and 7. 


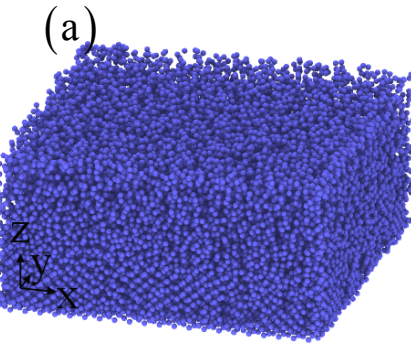

(e)

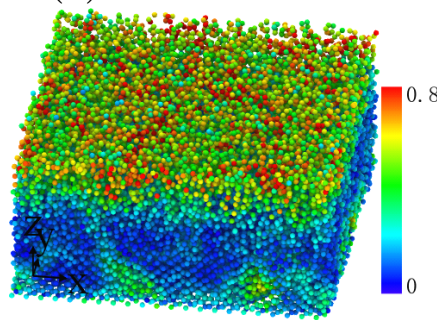

(b)

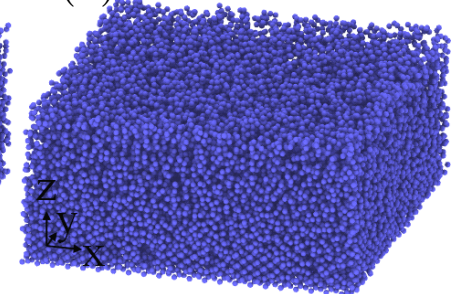

(f)

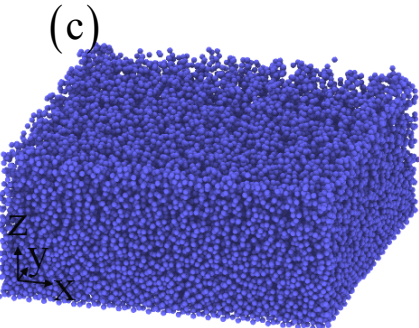

$(\mathrm{g})$

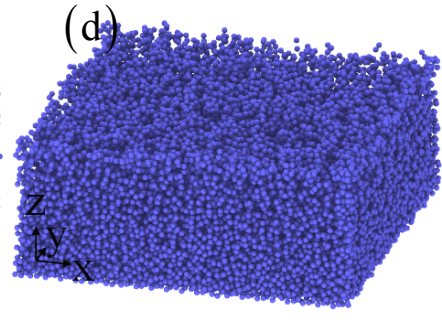

(h)
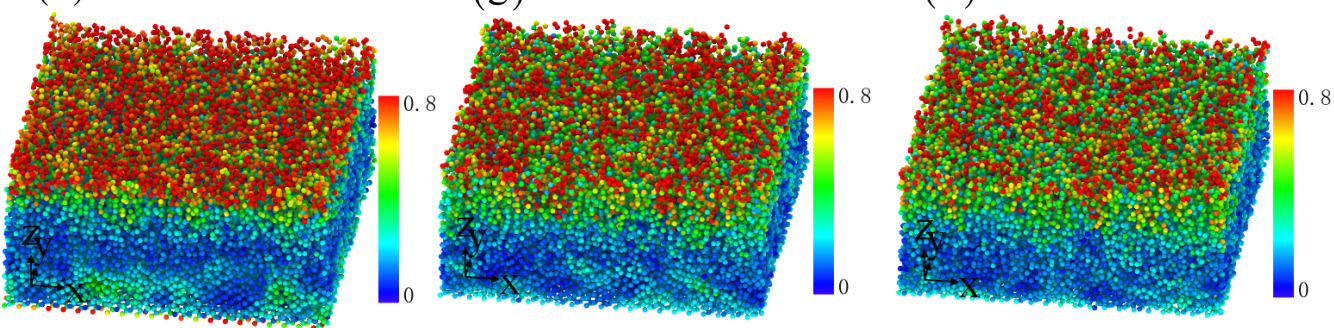

Fig. 14. The deformation behavior of Si electrode (a-d), and the strain distribution of Si electrode (e-f) at various temperatures: $100 \mathrm{~K}(\mathrm{a}, \mathrm{e}), 200 \mathrm{~K}(\mathrm{~b}, \mathrm{f}), 500 \mathrm{~K}(\mathrm{c}, \mathrm{g})$, and $800 \mathrm{~K}(\mathrm{~d}, \mathrm{~h})$.

(a)

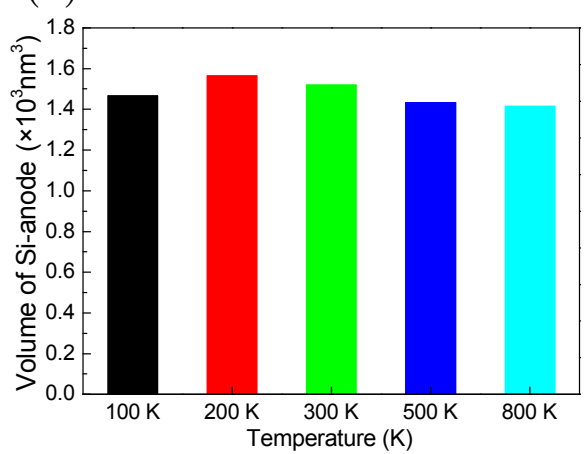

(b)

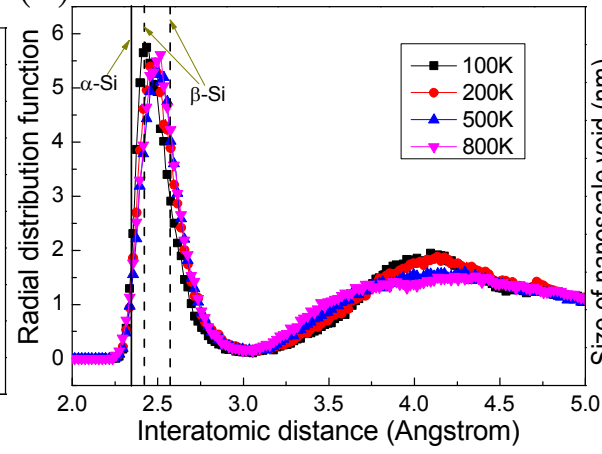

(c)

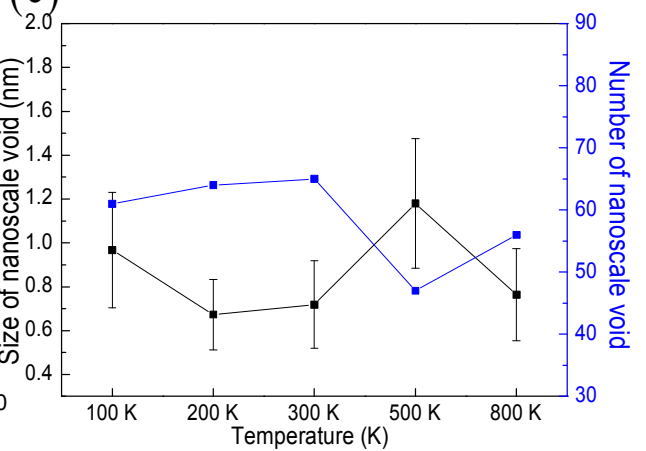

Fig. 15. The volume of Si electrode (a), RDF showing interatomic Si-Si bond length (b), and the size and number of nanoscale void generated at surface of Si anode (c) at various temperatures.

(a)

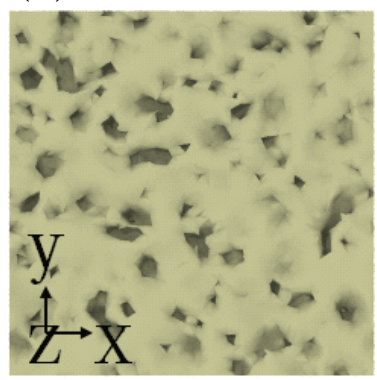

(b)

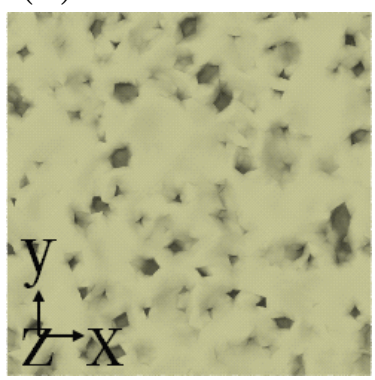

(c)

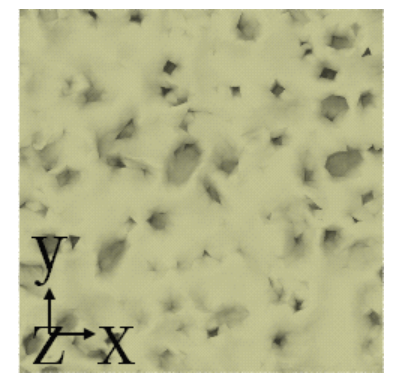

(d)

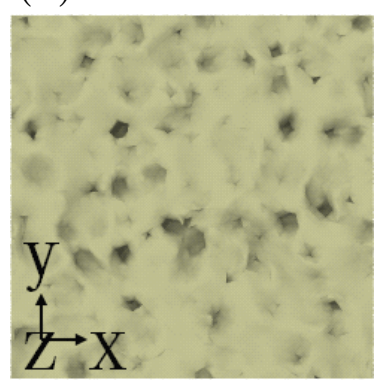

Fig. 16. The surface morphology of Si electrode at various temperatures (a-d): $100 \mathrm{~K}, 200 \mathrm{~K}, 500 \mathrm{~K}$, and 800 K. 
(a)

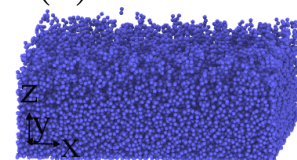

(e)

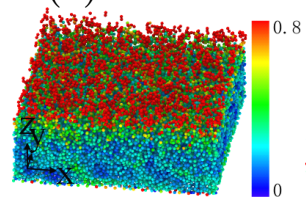

(b)

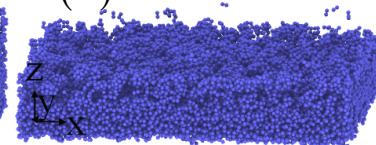

(f) (c)

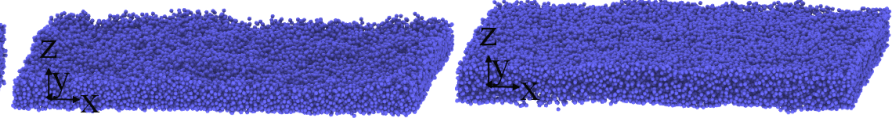

(g) (d)

(h)
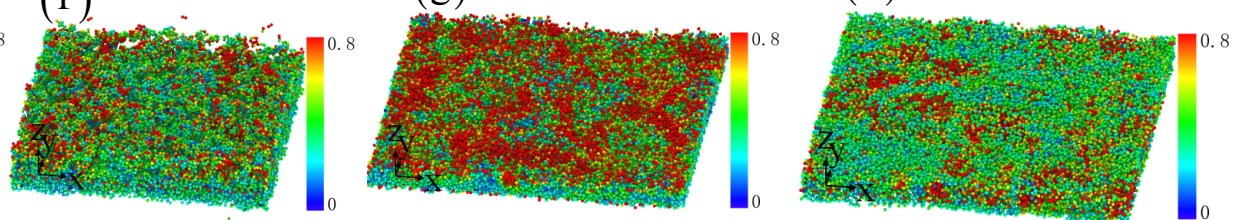

Fig. 17. The deformation behavior of $\mathrm{Si}$ anode (a-d), and the strain distribution of $\mathrm{Si}$ electrode (e-h) at

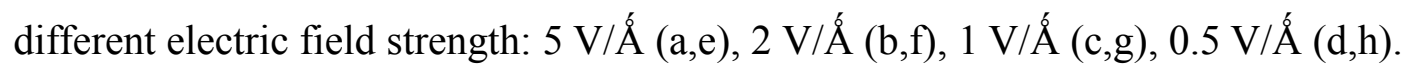
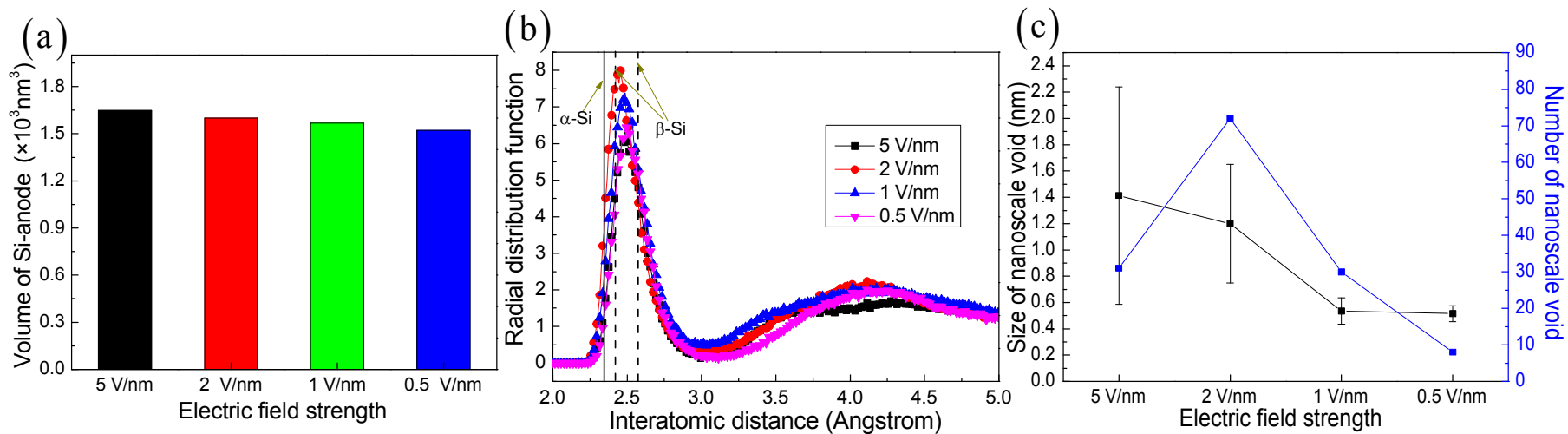

Fig. 18. The volume of Si electrode (a), RDF showing interatomic bond length (b), and the size and number of nanoscale void generated at surface of Si anode (c) at different electric field strength.

(b)

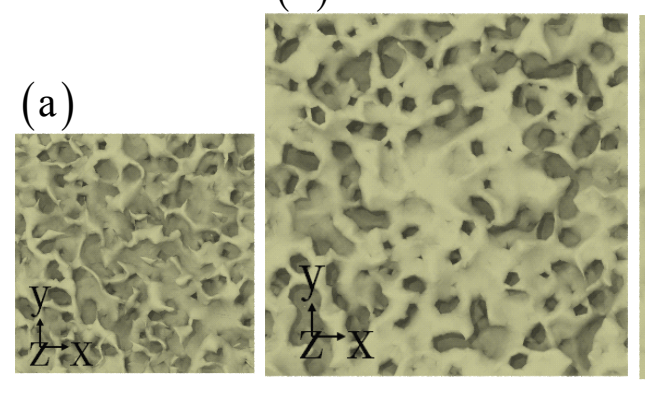

(c)

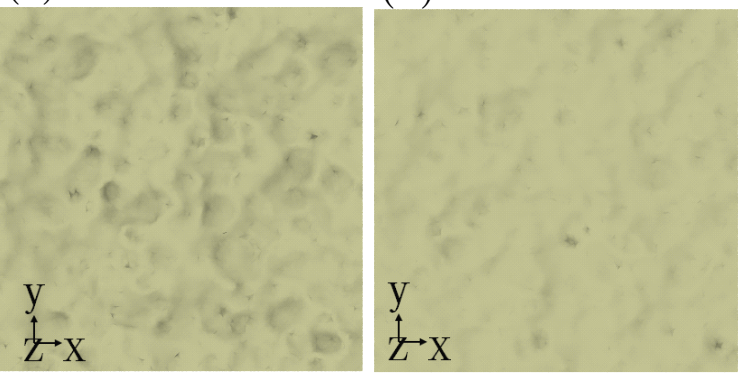

Fig. 19. The morphology of Si electrode at different electric field strength (a-d): $5 \mathrm{~V} / \hat{A}, 2 \mathrm{~V} / \hat{A}, 1 \mathrm{~V} / \hat{A}, 0.5$ V/Á. 

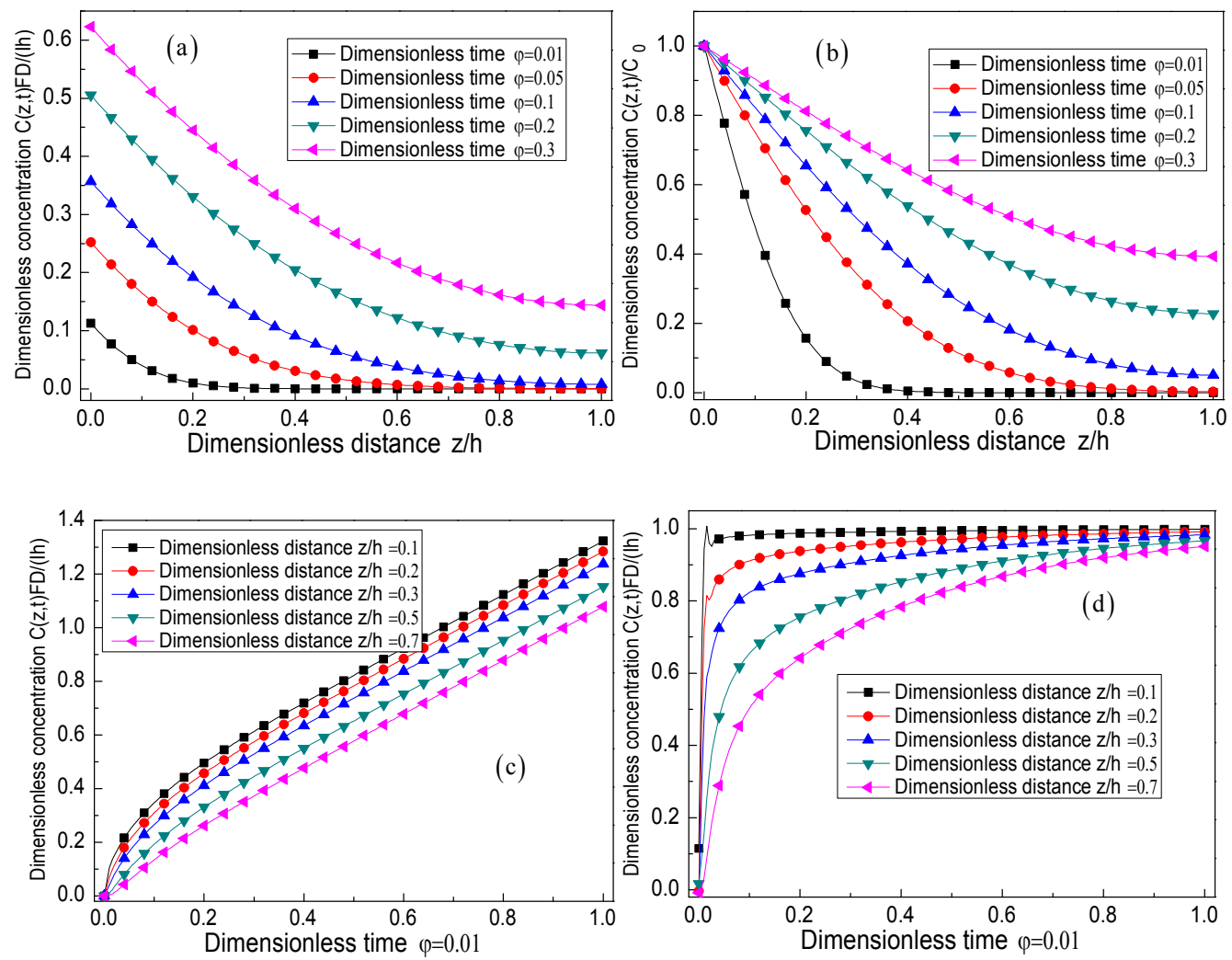

Fig. 20. Dimensionless concentration with increasing distance under galvanostatic operation (a) and under potentiostatic operation (b). Dimensionless concentration with increasing time under galvanostatic operation (c) and under potentiostatic operation (d). 

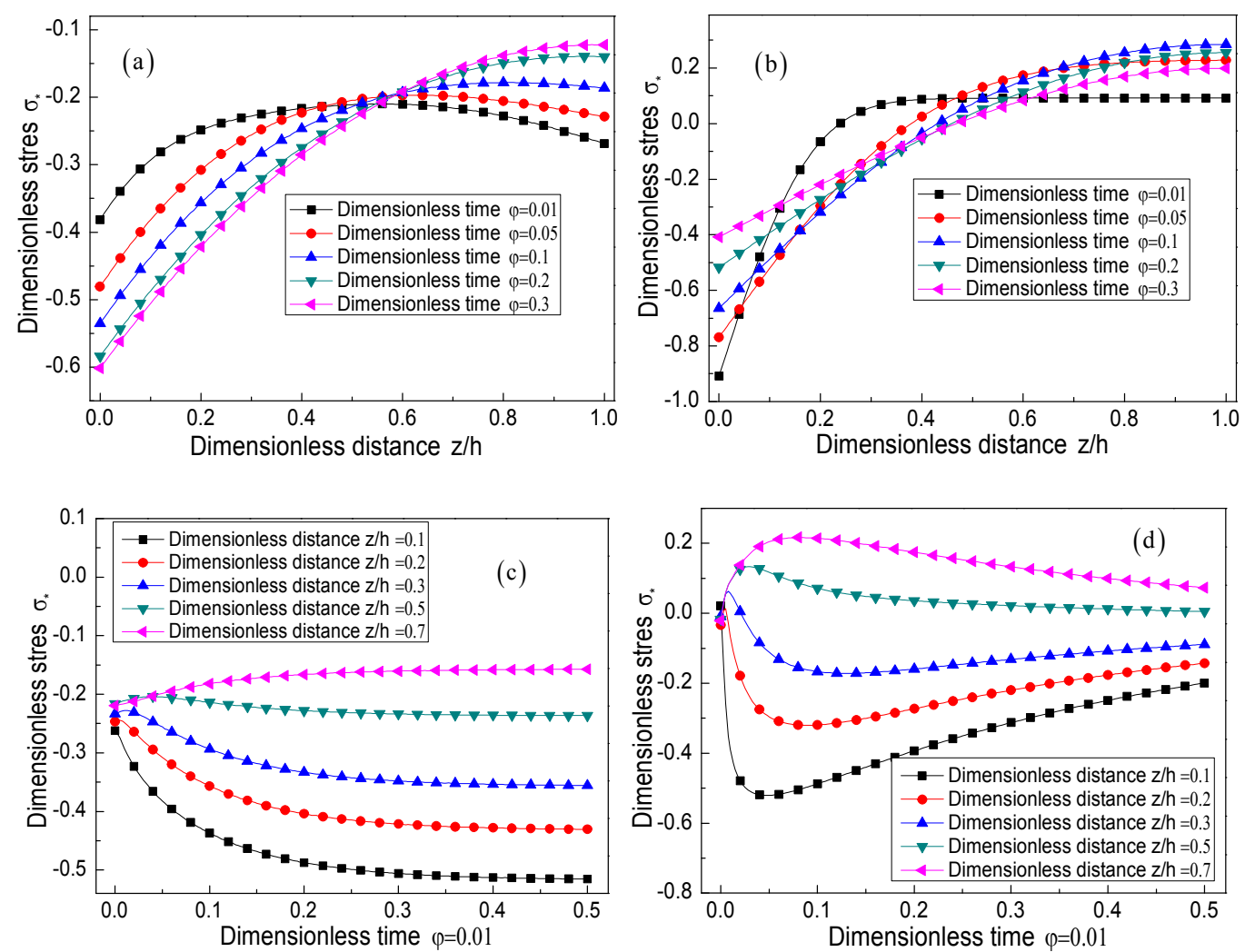

Fig. 21. Dimensionless biaxial stress with increasing distance under galvanostatic operation (a) and under potentiostatic operation (b). Dimensionless biaxial stress with increasing time under galvanostatic operation (c) and under potentiostatic operation (d). 